\title{
Heterogeneous Nanostructure Array for Electrochemical Energy \\ Conversion and Storage
}

Min Zhou ${ }^{1}$, Yang Xu ${ }^{1}$, Yong Lei ${ }^{*}$

Institute of Physics \& Institute of Macro- and Nanotechnologies® (IMN), Ilmenau University of Technology, Ilmenau 98693, Germany.

*Corresponding author. E-mail address: yong.lei@tu-ilmenau.de

${ }^{1}$ Dr. M. Zhou and Dr. Y. Xu contributed equally to this work.

Keywords: heterogeneous nanostructure array, electrochemistry, energy conversion, energy storage, device design.

\begin{abstract}
Rapid development of modern society raises more and more requirements for highly efficient energy conversion and storage. Electrochemical devices stand out as a most viable option for eventual substitute for fossil fuels, but suffer from problems like durability, operability, etc. Heterogeneous nanostructure arrays with distinguished superiorities have thus attracted intensive attention and yielded favorable electrochemical performance. In pursuit of deep understandings of their working modes, this review will focus on the interconnection among different constituents within each individual unit to correlate microscopic electrochemical processes with macroscopic performance. Here, the motivation of employing heterogeneous nanostructure arrays is first summarized. Then, the design principles, including three working modes, 'Function-Function', 'Function-Assistance', 'Single-unit device', are analyzed comprehensively to illuminate the interconnection among different constituents in electrochemical energy conversion and storage processes. Solar water splitting (energy conversion), alkali-ion battery and supercapacitors (energy storage) are termed collectively as typical electrochemical energy technologies to illustrate the
\end{abstract}


superiorities of heterogeneous nanostructure arrays. Finally, perspectives of related fields will be concluded to broaden the future of heterogeneous nanostructure arrays in future. 


\section{Introduction}

Energy has been regarded as a central societal issue with tremendous impacts on global economy, environment and our daily life [1-6]. With rapid development of modern society and increased population, the annual worldwide energy consumption is estimated to be 46 trillion watts by the end of twenty-first century. At present, most energy supply comes from fossil fuels, like oil (35\%), coal $(23 \%)$ and natural gas (21\%) [7]. Such over-reliance results in deterioration of climate and environment. Limited resources cannot satisfy the ever-increasing energy requirements. It is essential to develop novel technologies on conversion and storage of renewable energy, which would be expected to be more practical and price-competitive with fossil fuels in future. Electrochemical energy conversion and storage technologies can convert energy into widely-used and practicable chemical energy or electrical energy, and store/release them replying on common redox electrochemical principles with little or no pollution. Hence, they stand out as most viable option for eventual [8]. Although rapid development of research in this area, corresponding commercialization suffer from unsatisfied performance, such as durability, operability etc $[1,2,9]$.

Generally, innovation of materials lies at the heart in pursuit of further breakthroughs in electrochemical devices. Present commercial devices are mainly constructed by planar configuration $[10,11]$, remaining much room for approaching the theoretical capabilities of energy conversion and storage. To break this obstacle, heterogeneous nanostructure arrays, i.e. large-scale alignment of oriented nano-units that compose of more than one constituent on the substrate, have attracted intensive attention and exhibited excellent superiorities over the conventional counterparts. First, heterogeneous nanostructure arrays contain the tunable units in nano-scaled dimensionality, which possess unusual physical and chemical properties due to the confining effects and large surface-to-volume ratio. The combination of surface and bulk properties to the entire behaviors is beneficial during electrochemical processes 
[1]. Second, the unit with fixed spatial orientation and high regularity can be assembled into large-scale array alignment and realize electrochemical energy conversion and storage at nanoscale independently without interrupting the electrochemical reactions in other units $[6,9,10,12]$. It is of significance to investigate electrochemical processes macroscopically for further microscopic optimization of energy conversion and storage devices. Third, heterogeneous nanostructure arrays, compared with homogeneous architectures, combine the advantages of different constituents and achieve the synergistic (" $1+1>2$ ") performance through the strong interactions among different constituents [13]. Such material basis thus offers a versatile platform to potentially push forward the research on electrochemical energy conversion and storage, and further intrigue the design of single unit device electrode with multifunction.

As known, properties of an ensemble of arrayed heterogeneous nanounits are intimately connected to the designed constituents and their interconnections within and among each individual unit. Despite the electrochemical devices differ from each other, the fundamental principle of construction is similar. Looking through previous reports on heterogeneous nanostructure arrays, we find that there are three basic working modes according to the roles in the final electrochemical devices. The first mode called 'Function-Function', where different constituents play the same functional role at one time. If one constituent possesses the main electrochemical function, while other constituents can adjust related properties of functional constituent to assist the main function, this mode can be regard as 'Function-Assistance'. In both modes, it is worth mentioning that heterogeneous nanostructure arrays complete only one function, and additional functional constituents are also necessary to complete the entire circuit of the final electrochemical devices. Keeping this in mind, researchers also devote efforts on 'Single-unit device', where each unit serves as an independent electrochemical device. Within single unit, different constituents with separated functions can work together 
to complete the construction of final device in nanoscale.

Although there have been a few review articles $[2,4,6,8,10,11,14,15]$ concerning the electrochemical energy conversion and storage based on nanostructured arrays, most of them prefer to the structural features or fabrication processes and are solely dedicated to the interconnection among different constituents. To fulfill this gap, in this review, we focus on the progress of heterogeneous nanostructure arrays for electrochemical energy conversion and storage from the viewpoint of the intrinsic relationships among various constituents. First, the motivation of employing heterogeneous nanostructure arrays is summarized for electrochemical devices. The construction, fabrication progress, structural features and resulting superiorities are also briefly reviewed. Then, the design principles, including three working modes: 'Function-Function', 'Function-Assistance', 'Single-unit device', are analyzed according to the detailed roles in electrochemical processes. Due to space limitations, it is beyond the scope of this review to give an exhaustive summary of all the electrochemical energy conversion and storage devices benefited from heterogeneous nanostructure arrays now or in future. Solar water splitting (energy conversion), alkali-ion battery and supercapacitors (energy storage) are termed collectively as typical electrochemical energy technologies to illustrate the superiorities of heterogeneous nanostructure arrays. Finally, perspectives of related fields will be concluded to broaden the future about where and how the heterogeneous nanostructure arrays can be used in energy area.

\section{Motivation of heterogeneous nanostructure arrays for electrochemical energy conversion and storage}

\subsection{Construction of heterogeneous nanostructure arrays}

Heterogeneous nanostructure arrays (HNAs) comprise a relatively new, yet already immense area towards defined functionality [13]. Tracing the previous progress of HNAs, the constructions with binary constituents have been summarized in Scheme 1 
as typical examples. According to the types of interconnection interfacial between the two constituents, binary HNAs can be classified into three categories: (1) fully interfacial contact, (2) quasi-facet interfacial contact, (3) non-contact.

\section{Fully interfacial contact}
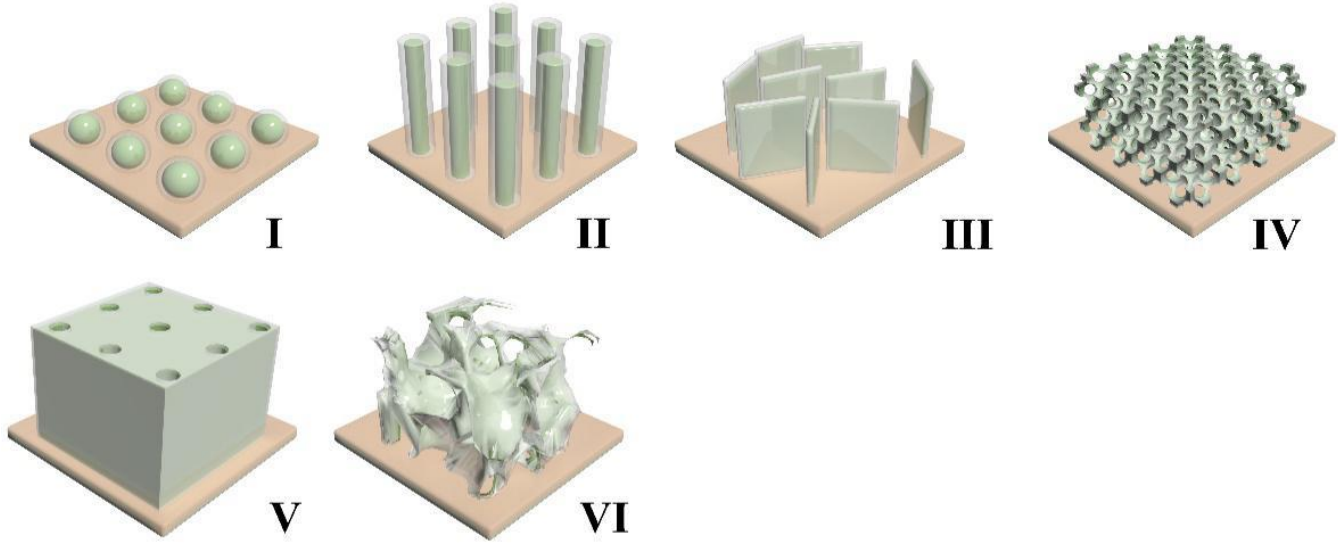

\section{Quasi-facet interfacial contact}
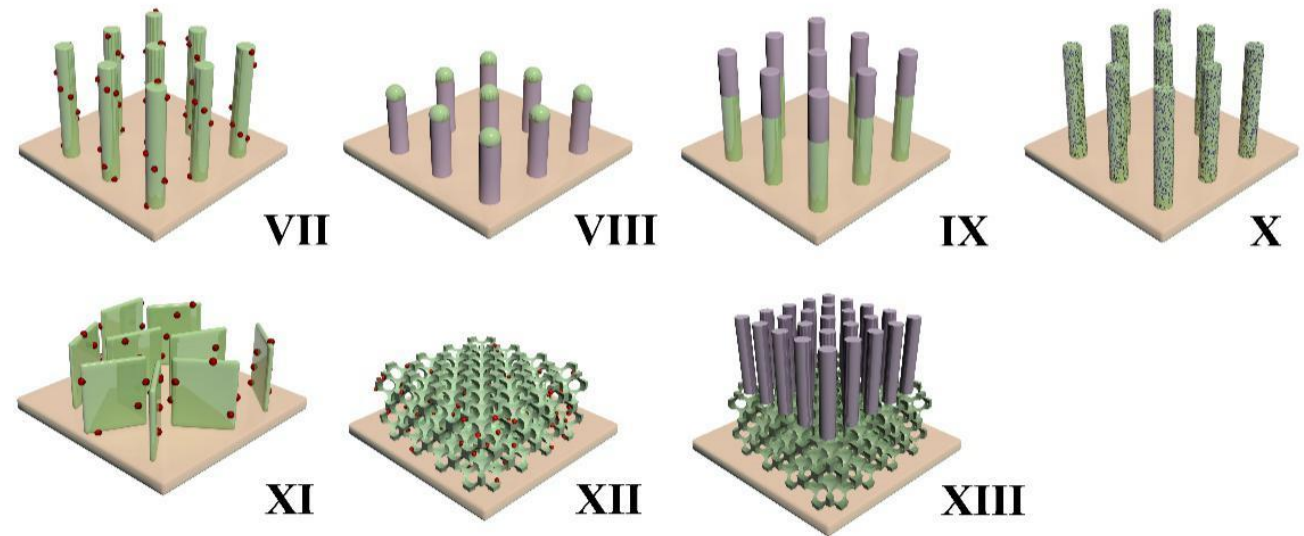

Non-contact
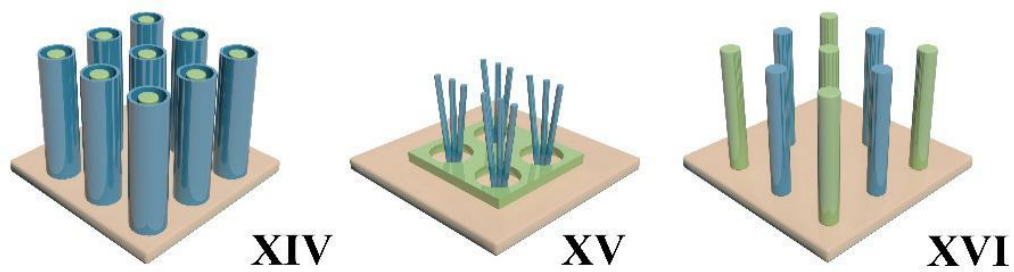

Scheme 1. Schematic illustration of the typical geometries of binary heterogeneous nanostructure arrays for electrochemical energy conversion and storage according to the interconnection way between the two constituents.

The first category is defined because of the existence of fully interfacial contact (I-VI). One constituent acts as the skeleton, and while the other constituent is 
deposited completely on the skeleton to form a continuous and conformal film with additional activation, passivation, stabilization, or physical/chemical functionality. In this sense, the first skeleton constituent is completely protected from external environment. Sometimes, discontinuous coating is also designed to balance the contact area of the inner constituent with external environment and the functions of the outer constituent.

In the second category, generally, the quasi-facet interface will be formed between the two constituents. One constituent also serves as the skeleton, and the second constituent scatter either on partial surface randomly (VII, XI, XII) or at special location, such as the top (VIII, IX, XIII). Another special construction is that the second constituent is embedded into the first one homogeneously (X). Both constituents can tangible to the external environment with the presence of new kind of interfaces.

Most interestingly, the two constituents can be designed without direct contact. The second constituent can choose to locate either around the first constituent but remaining some gaps (XIV), or in the intervals of the first one (XV, XVI). This design allows for the similar external environment for different constituents, but respective independence to the most degree. The interfacial gap between constituents may create unpredictable profits toward overall performance.

Despite this classification is defined within binary HNAs, it is also universal for HNAs with multiple constituents, where more than one kind of interface is produced. Each interface can be formed with the choice from above-mentioned types. Either same category or hybridization of different categories holds the promise to create multiple interfaces toward complex functionalization.

\subsection{Fabrication strategy of heterogeneous nanostructure arrays}

The realization of this tentative idea of HNAs advances by the development of fabrication strategies in a large yield. Considering the applications in electrochemical 
energy conversion and storage, all of these HNAs are designed to directly grow on the conductive substrates, such as fluorine-doped tin oxide (FTO), tin-doped indium oxide (ITO), Cu, Al, Ni, Ti, stainless steel, carbon cloth, etc [11]. The unit of such array architectures can be constructed in the form of either 0D nanodot, 1D nanorod/nanowire/nanotube, 2D nanosheet and 3D porous/branched/framework. An amount of fabrication techniques, including versatile wet-chemical approaches, photo-/electro-/magnet-assisted deposition, vapor-phase deposition, atomic layer deposition, pyrolysis, sputtering, dry/wet etching, plasma treatment, etc. have been used to realize the heterogeneous architectures. Due to the distinctiveness nature of heterogeneous architectures, the fabrication strategies can be summarized by the growth sequence of different constituents into two main kinds: fabrication in sequence and fabrication in simultaneity. Corresponding fabrication strategies are visualized as diagramed in Scheme 2.
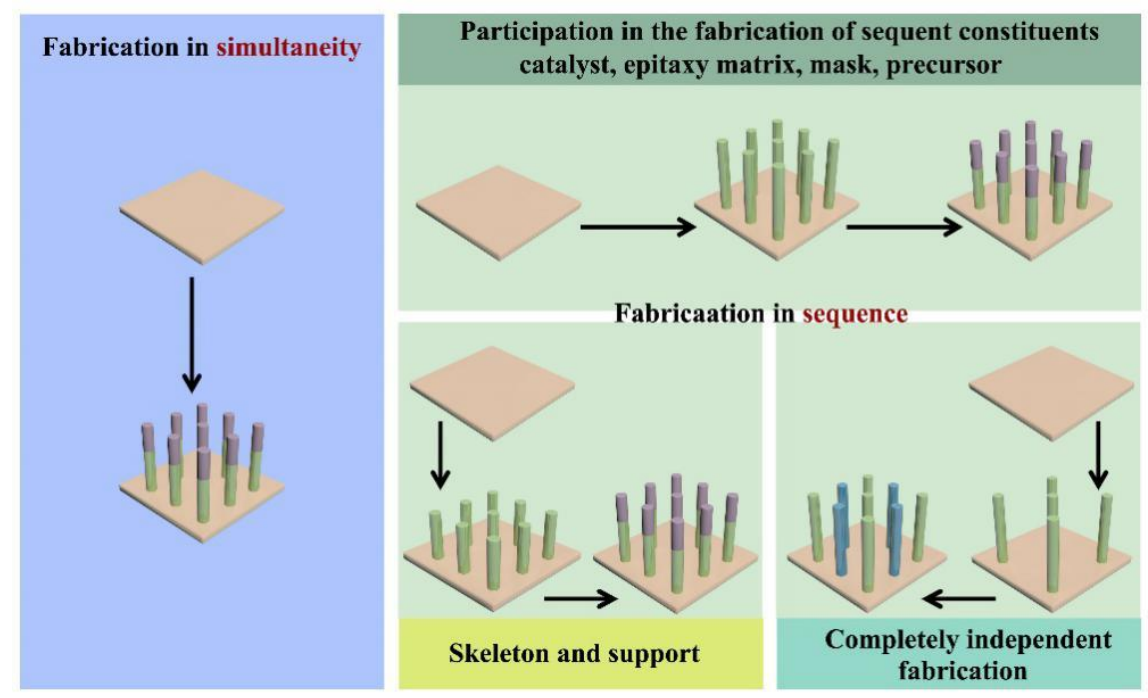

Scheme 2. Schematic of fabrication strategies for heterogeneous nanostructure arrays according to the growth sequence of different constituents: fabrication in simultaneity and fabrication in sequence. Considering the different roles of pre-formed constituents on the growth of other constituents, fabrication in sequence can be further classified into three categories. The pre-formed constituents can participate in the fabrication of sequent constituents as catalyst, epitaxy matrix, mask or direct precursor, and while 
will not directly participate in the fabrication of sequent constituents, serving as only skeleton and support. Furthermore, fabrications of different constituents can be completely independent.

Fabrication in simultaneity is defined as the strategy that heterogeneous constituents are produced at the same time. In general, the precursors of different constituents are first pre-designed with special morphology and relative position based on templates. For example, CdS-ologo(phenyleevinylenes) (OPVs) hybrid nanorods [16] can be formed by immersing AAO templates into CdS and OPVs mixtures under reduced pressure. Besides, one-step natural formation of heterogeneous nanostructure arrays can be directly completed on the substrate when all precursors are mixed together. GaN/BN core/shell nanocables are synthesized on Si substrate used a one-step thermal CVD reaction of $\mathrm{GaP}, \mathrm{B}_{2} \mathrm{O}_{3}$, and $\mathrm{NH}_{3}$ [17]. $\mathrm{Si} / \mathrm{CdSe}$ core-shell nanocables are available on the Si substrate by a simple one-step thermal evaporation of CdSe powder with the presence of oxygen [18].

Fabrication in sequence is one of the most frequently-used strategy for HNAs with more possibilities for design. One nanostructure arrays can first grow on the substrates by a series of accessible techniques, including natural growth, etching, and template-induced growth [6, 10-12, 19] During this step, the pre-formed constituent always plays different roles on the growth of other constituents in the following three categories. (1) it can serve as catalysts or epitaxy matrix [20] to facilitate the growth of other constituents, or stand as masks for the subsequent etching, as well as natural growth techniques. Direct participation in the growth is also promising, where the first constituent will transform to other constituents as precursors by means of self-sacrifice template approaches, electrochemical anodization [21], anchoring functional groups [22], surface doping [23], etc. A typical example is $\mathrm{Fe}_{2} \mathrm{O}_{3} / \mathrm{Fe}_{3} \mathrm{C}$-graphene heterogeneous arrays that can be constructed by integrating graphene into pre-anodized $\mathrm{Fe}_{2} \mathrm{O}_{3}$ nanoporous arrays by chemical vapor deposition (CVD). $\mathrm{Fe}_{3} \mathrm{C}$ phase will be formed in subsequent CVD post-annealing process by 
reactions between partially $\mathrm{Fe}_{2} \mathrm{O}_{3}$ and graphene [24]. (2) the pre-formed constituent can be straightly utilized as growth skeleton and support for subsequent constituents. All the above-mentioned categories of heterogeneous architectures are thus possible in turn. Such an array under nanometer scale possesses extremely high surface ratios, allowing for the potential to build multisegmented striped architectures or hierarchical architectures with diverse dimensional constituents [25]. It is also facile to form continuous or discontinuous coatings layer by layer to construct coaxial solid core-shell architectures as shown in Scheme 1 (I-VI) or partial modifications to realize quasi-facet interfacial contact (Scheme 1, VII-XIII). As examples, various well-aligned metal-organic frameworks (MOF) hybrid arrays can be realized by means of integrating MOF growth directly on different oxide nanoarrays, such as CoO@ZIF-67 nanorod array, CoO@Co-MOF-74 nanorod array, NiO@Ni-MOF-74, ZnO@ZIF-CoZn core-sheath nanowire array, CoAl-LDH@ZIF-67 nanosheet array, $\mathrm{Ni}_{3} \mathrm{~S}_{2}$ nanorod- Co-MOF-74 porous membrane, etc [28-31]. If some constituents would be removed later, hollow core-shell architectures are highly expected [26, 27]. Apart from these close interconnections, completely independent fabrication of different constituents is also promising by the assistance of templates or electrode design. For example, Wen et al. developed novel binary pore anodic aluminum oxide (AAO) templates [32], providing feasible and independent choices of growth techniques and parameter optimization of different constituents.

It is worth mentioning that fabrication in sequence can contain several steps with strategies of fabrication in simultaneity to construct some constituents in one step and other constituents in the rest steps, offering more possibilities for the choice and combination of modulated constituents and interfaces. As for materials, existing HNAs have combined metals ( $\mathrm{Au}, \mathrm{Ag}, \mathrm{Ni}, \mathrm{Cu}, \mathrm{Pt}$, etc.), semiconductors (oxides, sulfides, nitrides, carbides, phosphide, hydroxide, oxysulfide, oxynitride, $\mathrm{C}_{3} \mathrm{~N}_{4}, \mathrm{Si}$, etc.), carbon (amorphous carbon, hard carbon, carbon nanotube, graphene, carbon quantum dots, etc.), polymers (OPVs, polyaniline, polypyrrole, 
poly(3,4-ethylenedioxythiophene), etc.), amorphous materials and other functional groups. Such diversity and resulting superiorities from distinct structural features make HNAs more competitive toward the requirements of electrochemical energy conversion and storage.

\subsection{Structural features and superiorities of heterogeneous nanostructure arrays for electrochemical energy conversion and storage}

Although the devices of electrochemical energy storage and conversion differ from field to field, they all involve basic physical interactions and/or chemical reactions both at the surface or interface and within the active materials. One of the fundamental challenges is to improve generation, transport and utilization of electrons, holes, ions and other molecular species for electrochemical processes [7, 11], raising complex requirements, like the optical, electrical and chemical properties, for material design. Heterogeneous nanostructure array stands out as a promising architecture to meet this fundamental challenge due to their distinctive structural features:

(1) Units at nanoscale

In a given unit, it is common-sense that at least one dimension has been reduced to a nanometer size spanning from either $0 \mathrm{D}, 1 \mathrm{D}, 2 \mathrm{D}$ to $3 \mathrm{D}$. The in-depth consideration of physical and chemical processes that occur on nanometer scales is the defining principle guiding advances in electrochemical energy conversion and storage technologies [33].

First, units at nanoscale contribute primarily to the tunable light harvesting, which is benefit for solar conversion devices. According to the degree of confinement in nanoscale, the band gap of materials may become wide with decreased size and dimensionality (i.e. increased number of confined dimensions) [34-36]. Similar trends can also be found in different material systems, like oxides [37], chalcogenides [38, 39], nitride [40, 41], Si [42, 43].

Second, nanoscale units will exert influences on charge migration and ion transport 
based on the tunable size and dimensionality. (i) With decreased size, the edge of conduction band (CB) will shift to more reducing potentials, while the valence band (VB) edge will turn to be more oxidized. On the basis of Marcus-Gerischer theory, the rates of interfacial charge transfer and reactions may be fastened owing to the increased thermodynamic driving force [44-46]. (ii) Materials with different size and dimensionality show distinctive ability for charge migration and ion transport. For example, the delithiation time of $\mathrm{LiFePO}_{4}$ nanoparticles $(100 \mathrm{~nm})$ is much less than a second $(0.01 \mathrm{~s})$, the value of which is in a second in micrometer-size particles $(1 \mu \mathrm{m})$ if intrinsic $\mathrm{Li}^{+}$mobility was rate-controlling [47]. The electron mobility at room temperature in 2D graphene is in excess of $15000 \mathrm{~cm}^{2} \cdot \mathrm{V}^{-1} \cdot \mathrm{s}^{-1}$ [48], while the reported value is only around $5 \mathrm{~cm}^{2} \cdot \mathrm{V}^{-1} \cdot \mathrm{s}^{-1}$ for carbon black [49]. Another typical example is that the recombination lifetime at the band gap $(585 \mathrm{~nm})$ in 1D CdS nanorods (50 ps) are longer than that of $0 \mathrm{D}$ nanodots ( 2 and $36.1 \mathrm{ps}$ ) due to reduced optical phonon coupling during the recombination process of electrons and holes [50].

Third, decreased size results in increased number of atoms on the surface, which are incompletely bonded within the crystal lattice with disrupted crystalline periodicity and leave dangling orbital pointed outward from the crystal. This is double-edged for electrochemical process. More reactive sites can be thus created as well as trapping sites at the interfaces $[45,51]$.

Forth, units at nanoscale will offer opportunities for new mechanism energy storage. Poizot et al. observed that the superior long-term stability and high capacity during fast charging/discharging in nano transition metal oxide $\mathrm{MO}(\mathrm{Co}, \mathrm{Ni}, \mathrm{Cu}, \mathrm{Fe})$ come from the formation and decomposition of $\mathrm{Li}_{2} \mathrm{O}$ together with the reduction and oxidation of metal nanoparticles, which is totally different from classical intercalation, conversion and alloying mechanisms [52].

Many successful examples have proved the advantages of nanoscaled units. The ability of solar water splitting of BiVO4 0D nanoparticles [53], 1D nanopyramids [54], 2D nanowalls [55] and 3D ordered macroporous structure (3DOM) [56-58] show the 
strong dependence and tunability on the size and dimensionality. Corresponding lithium or sodium storage ability of $\mathrm{TiO}_{2}$ with different dimensionality, including 0D nanoparticle [59], 1D nanowire/nanotube [60,61], 2D nanosheet [62, 63], 3D porous structures[64, 65], all fully evidence the advantages of nanounits over bulk materials.

(2) large-scale array alignment

The outward overall performance depends on not only the properties of nano-units, but also the way to integrate them. Of even greater appeal, particular attention has been paid to large-scale array alignment with fixed spatial orientation and high regularity.

First, materials with a refractive-index contrast regularly patterned on the order of the wavelength of light strongly modulate light of certain frequencies [51]. The electric field distribution scope is thus greatly enhanced compared with that of planar film. Hence, extra light harvesting induced by the array structure appears by means of light scattering, antireflection, etc, and contributes to improved solar conversion efficiency. For instance, periodic dielectric design has been introduced into $\mathrm{Bi}_{2} \mathrm{WO}_{6}$ nanowall units to lengthen the light-matter interaction path based on the multiple reflections and scatterings at the interfaces. Enhanced solar conversion efficiency is achieved in return for this design [66]. Employing the design of photonic crystal is also able to greatly improve light harvesting and corresponding solar conversion efficiencies. Successful examples have been found in diverse material systems, such as $\mathrm{Si}, \mathrm{Ge}, \mathrm{TiO}_{2}, \mathrm{WO}_{3}, \mathrm{CdS}$, etc.

Second, the units are arranged into array alignment with adjustable interval space. (i) The open-edge geometry is beneficial for increasing the contact between the materials and electrolyte. It thus offers more possibilities for easy electrolyte accessibility and fast charge and/or ion diffusion from electrolyte to reach inner region of units, and vice versa. The active sites at the material/electrolyte can be also maximized. Extended band bending formed at the interface gives rise to the depletion region where charges yielded by incident photons can be separated and facilitates the 
electrochemical reactions at the interface [56]. (ii) The existence of interval space is in favor of elimination of produced gas from surface and serves as a structural buffer zone to accommodate the strain of ion inserting and tolerate the volume expansion. Some deterioration problems with repeated illumination or charge/discharge cycles, like pulverization, agglomeration, exfoliation, are able to be addressed in some degree, enabling retaining stability after long-time operations.

(3) Heterogeneous innovation of each unit

Notwithstanding the intense research efforts on electrochemical energy conversion and storage, the lack of suitable materials still remains as a key reason for sluggish progress. The ideal material must be efficient in generation, transport and utilization of electrons, holes, ions and other molecular species. Unfortunately, such a material has yet to be found $[67,68]$. The difficulties in finding a suitable material stem from complex, sometimes conflicting property expectations [69]. The adjustment in size, dimensionality and alignment aims at optimizing the properties and alleviating this reliance to materials, however, the opportunity for tunability is still limited because of competing nature of materials' intrinsic properties. Like all the other ties, new challenges come out along with the advantages. For instance, large band gap is enough to drive redox reactions, but reduces light harvesting. Large surface-volume ratio can fasten the surface reactions but offer more sites for surface charge recombination and reduce space charge layer thickness with increased difficulties of charge separation in solar water splitting. These considerations underscore how the difficulties in finding a single material emerge to satisfy all the requirements for optimized electrochemical processes. Hence, our goal is to identify problems and come up with solutions by heterogeneous constituent innovation, the overall properties of which are attributed to individual and collective contributions from different constituents, as well as from the interfaces among the constituents. Each constituent can be tailored independently based on deep understanding of the influences from structural feature (1) and (2) to satisfy more demands. Multiple 
constituents complement each other in a reciprocal way to generate additional or completely new benefits and synergetic effects, which are not available in single unit. The interfacial region may afford unpredictable profits upon potential formation of junctions or interaction in certain distance. As a result, absorbed range in solar spectrum can be broadened by means of multiple light absorbers. Band gap engineering can be used to solve the problems from reduced charge separation. The issues regarding surface charge recombination can be addressed by changing the surface kinetic by coating other constituents. If all the nanoscale constituents directly connect with highly conductive composite, charge transport is expected to be accelerated over macroscopic distance during the processes of electrochemical energy conversion and storage. All these interactions exhibit excellent future for maximizing the efficiency during electrochemical processes and produce a ' $1+1>2$ ' optimization.

On the basis of above-mentioned recognitions, the three distinctive structural features of heterogeneous nanostructure arrays play vital roles on achieving high efficiency of electrochemical energy conversion and storage. Feature (1), (2), (3) provide innovation solutions from size/dimensionality, alignment and constituent. Feature (1) and (2) offer tunable material basis and morphology model according to the requirements for constituent innovation, so that feature (3) can realize integrated advantages with bonus from the constituent combination. The three features make HNAs possible for further fundamental researches and practical applications with the suitable design principle in electrochemical energy conversion and storage as elaborated in the following section.

\section{Design principle of heterogeneous nanostructure arrays for electrochemical energy conversion and storage}

To date, many efforts have been devoted on employing HNAs in electrochemical energy conversion and storage because of the inspiration of the three distinct features. Taking a panoramic view of previous literatures, subtle-designed heterogeneous 
architectures, especially based on Feature (3), are more multifarious and efficient in many cases for targeted optimization of material properties and corresponding influences on electrochemical steps. Researchers have tried to investigate HNAs from various perspectives, such as fabrication approach, constituent type, structural feature and application, etc. Few of literatures shift their focuses on the design principle related to heterogeneous functional interconnectivity, which clearly demonstrates the dependence between microscopic electrochemical processes and macroscopic performance parameters. In order to exploit the structural advantages of HNAs to the full, it is of great significance to establish an understanding bridging the gap between overall performances of entire ensembles and fundamental heterogeneous functional interconnectivity in nano-region. Intrinsic design and relationships among various constituents within nanostructured arrays indisputably pioneer the development of further design of HNAs.

We have shown above a large diversity of HNAs with various combinations of constituents and interfaces as well as highly controllable geometric characteristics. Standing on such abundant material basis with heterogeneous innovation (Feature 3), we find that the design principle for electrochemical energy conversion and storage can be mainly classified into three working modes among constituents: 'Function-Function', 'Function-Assistance' and 'Single-unit device' as shown in Scheme 3.

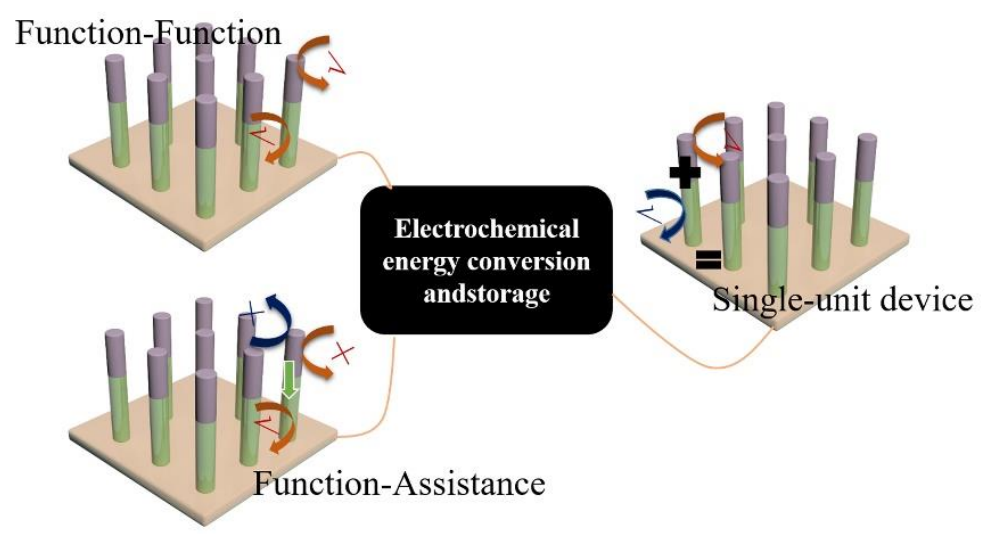

Scheme 3. Three promising working modes of heterogenerous nanostructure arrays in 
electrochemical energy conversion and storage.

For 'Function-Function' mode, different constituents perform the same functionality at one time and the overall property relies on their combined actions. More specifically, each constituent holds the capability of energy conversion or storage and directly participate related electrochemical steps.

For 'Function-Assistance' mode, one constituent ('functional constituent') dominates the overall performance, while the other constituent ('assistance constituent') will play an assistance role instead of joining all the electrochemical approaches directly. It will adjust the properties of functional one through certain types of physical or chemical influences. (e.g., electric field, magnetic field, chemical environment, light reflection and scattering, interfacial electronic structure, etc.). By changing these factors of the assistance constituent, information of the reflection from the stimuli can be obtained from the functional constituent, hence to control and improve its functions.

For 'Single-unit device', each constituent in one unit has its own function and dominates corresponding performance. Different constituents with separated functions cooperate with each other to realize the construction of final device within nanoscale. It means that each individual unit acts as a complete device in nano scale and achieves overall performance without any external assistance.

Such design principle with three working modes ranging from nano-scale units to macro-scale integration devices will indisputably pioneer the development of miniaturized multifunction devices. The comprehensive instructions will be elaborated in the following sections within the detailed application field of electrochemical energy conversion and storage.

\section{Application of heterogeneous nanostructure arrays for electrochemical energy conversion}


In general, electrochemical energy conversion includes many applications, like solar water splitting, water splitting by electrolysis of water, solar cell, dye-sensitized solar cell (DSSC), etc. In view of the abundant and renewable nature of solar energy and water resources, solar water splitting has been regarded as one of the most attractive technologies of electrochemical energy conversion. More importantly, solar water splitting covers all the typical basic electrochemical steps of light absorption, charge separation/migration and surface reactions, while other applications can only cover part or same steps. Considering the reduced similarity among the introductions of different applications, we chose solar water splitting as the example to illuminate the roles of HNAs in the field of electrochemical energy conversion.

\subsection{Function-Function}

Specifically speaking, with regard to 'Function-Function' mode for solar water splitting, the entire HNAs can produce either oxygen or hydrogen, and each constituent holds the ability of light absorption, charge separation and charge migration. The initial aim of employing 'Function-Function' design comes from the requirements of adequate light harvesting. In order to power the whole reactions, the energy difference between the hole and the electron energy level (i.e. total input energy from solar light) should be more than the sum of the standard free energy and activation energy of the water splitting reaction, ohmic loss and other overpotential [70]. When single constituent is concerned, the bandgap is required to be larger than $1.6 \mathrm{eV}$ or $2.0 \mathrm{eV}$ with additional kinetic overpotential consideration [71, 72]. However, materials with such a wide gap can only harvest light within UV range, which only makes up about $10 \%$ of the total light output of the Sun. That means the overall efficiency of solar to-chemical energy conversion would be low even with $100 \%$ quantum effieiency. It inspires us to turn our eyes onto HNAs based on the above-mentioned superiorities from the distinguished features. If the constituent with wide bandgap can be combined with a constituent with relatively narrow bandgap, photons of higher energies are absorbed due to narrow bandgap, and meanwhile 
photons in the long wavelength region can be captured by the wide bandgap co-absorbers. $\mathrm{TiO}_{2}$ is one of most widely-used materials for solar water splitting, but suffers from limited light absorption in UV range (3.2 eV for anatase, $3.0 \mathrm{eV}$ for rutile) [73]. Various efforts have been done in pursuit of absorption in visible light range and even near-infrared light range. Many $\mathrm{TiO}_{2}$ nanostructure arrays have been designed to obtain additional light absorption first, such as nanorod array, nanotube array, nanowire array, nanosheet array, 3DOM, etc, and then coated with other materials with narrower bandgaps, including Si, CdS, CdSe, CdTe, CdSe, $\mathrm{MoS}_{2}, \mathrm{ZnIn}_{2} \mathrm{~S}_{4}, \mathrm{In}_{2} \mathrm{~S}_{3}$, $\mathrm{FeS}_{2}, \mathrm{InP}, \mathrm{ZnO}, \mathrm{SnO}, \mathrm{Fe}_{2} \mathrm{O}_{3}, \mathrm{Fe}_{2} \mathrm{TiO}_{5}, \mathrm{ZnFe}_{2} \mathrm{O}_{4}, \mathrm{BiVO}_{4}, \mathrm{BiOCl}, \mathrm{BiFeO}_{3}$, etc. in the form of dot, rod, wire, sheet, uniform shell, etc. Typical example of such design can be found in well-ordered $\mathrm{CdTe} / \mathrm{TiO}_{2}$ nanowire arrays based on $\mathrm{AAO}$ templates as shown in Figure 1a. Integration of CdTe $(1.44 \mathrm{eV})$ shell has extended the absorption range of $\mathrm{TiO}_{2}$ to around $800 \mathrm{~nm}$, which fully covers the whole visible light range (Figure 1b). Hence, the enhanced light harvesting in visible light range results in largely improved photocurrent density (maximium $\sim 1.1 \mathrm{~mA} \mathrm{~cm}^{-2}$ in Figure 1c) [74]. $\alpha-\mathrm{Fe}_{2} \mathrm{O}_{3}$ and $\mathrm{Si}$ core-shell nanowire arrays can even collect photons in red and near-infrared region $(600 \mathrm{~nm}<\lambda<1100 \mathrm{~nm})$ [75]. Apart from band engineering, additional constituent introduction of geometrical optics-based elements is also effective to increase photon-matter interaction [66]. Unit-assembled arrays hold this potential owing to their fixed orientation and regular alignment as analyzed in section 2.3. The formation of a bilayer structure with $3 \mathrm{DOM} \mathrm{TiO}_{2}$ and $\mathrm{ZnO}$ nanowire arrays is an example with optical coupling (Figure 1d) not only from individual contributions from $\mathrm{TiO}_{2}$ and $\mathrm{ZnO}$, but also from the photonic stop bands (Figure 1e) with appropriate positions [76]. The resulting enhancement of photocurrent density can be found clearly in Figure 1f. Combining the superiorities of band engineering and structural features of HNAs, similar trend is universally applied on other active materials. Of more interest, bandgap engineering based on multiple constituents possess more diversity of the different combination of constituents, interfaces and structure features [77]. For instance, $\mathrm{ZnO}$ nanowires are first grown on $\mathrm{Si}$ microwires, 
and then sensitized with $\mathrm{CdS}$ and $\mathrm{CdSe}$ quantum dots and modified with $\mathrm{IrOx}$ quantum dots as diagramed in Figure 1g. Such a multiple hierarchical heterogenerous array is remarkably beneficial for light absorption from multiple-level bandgap engineering in Figure 5h, and realizes a 171 times enhancement in photocurrent density compared to that of $\mathrm{ZnO}$ nanowires on a planar substrate (Figure 1i) [78].
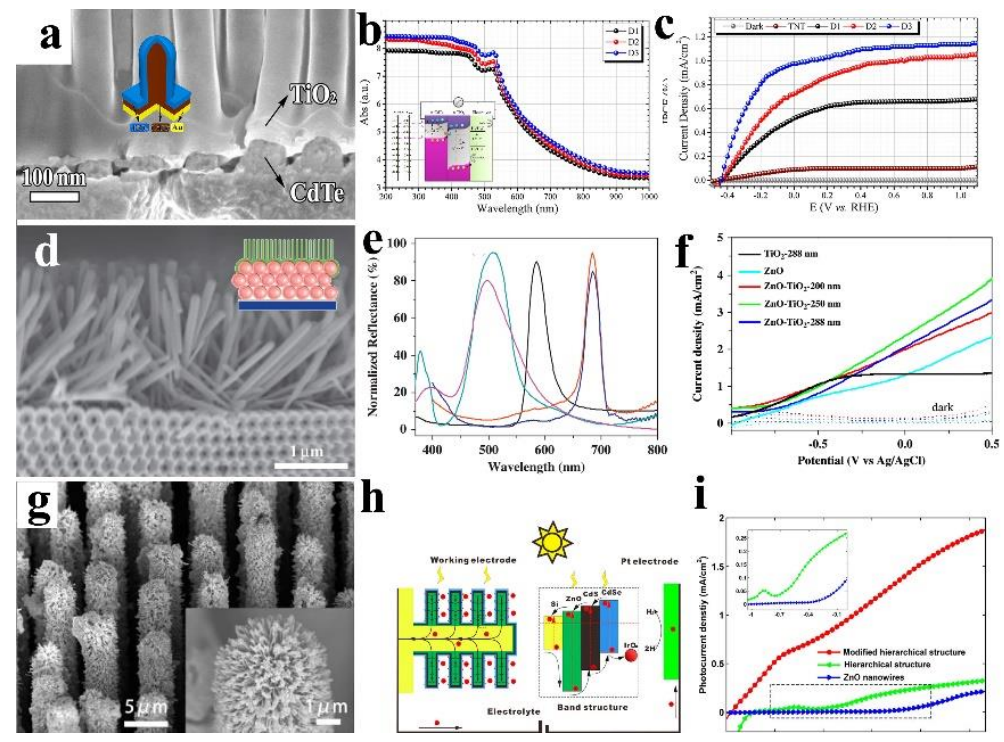

Figure 1. Typical examples of heterogeneous nanostructure arrays with 'Function-Function' working mode for enhanced light harvesting. (a-c) Cross-sectional view, absorption spectra and photocurrent density-potential curves of $\mathrm{CdTe} / \mathrm{TiO}_{2}$ nanowire array. Reproduced with permission. [74] Copyright 2016, Wiley-VCH Verlag GmbH \& Co. KGaA. (d-f) Cross-sectional view, absorption spectra and photocurrent density-potential curves of $\mathrm{ZnO} / \mathrm{TiO}_{2}$ nanowirethree-dimensionally ordered mesoporous (3DOM) array. Reproduced with permission. [77] Copyright 2012, Elsevier. (g) Titled view of the $\mathrm{Si} / \mathrm{ZnO} / \mathrm{CdS} / \mathrm{CdSe} / \mathrm{IrOx}$ hierarchical arrays, (h) Schematic of the water splitting process and energy level distribution of different constituents, (i) photocurrent density-potential curves of hierarchical arrays. Reproduced with permission. [78] Copyright 2014, American Chemical Society.

Among existing HNAs of 'Function-Function' mode, the functional constituents 
always directly contact between each other and form a heterojunction. Beside enhancement in light absorption, the appearance of an internal built-in field will force the migration of electrons and holes as illustrated in the band diagram of a heterojunction in Figure $1 \mathrm{~b}$ and $1 \mathrm{~h}$. Sometimes, electrons in $\mathrm{CB}$ of one constituent will be recombined with holes in VB of another constituent, resulting in some consumption of photo-conversion efficiency. Sometimes, electrons in $\mathrm{CB}$ of one constituent can be driven to the $\mathrm{CB}$ of another constituent, so do the migration of holes in VB. Fast separation of electrons and holes can suppresse charge recombination and promote photo-conversion efficiency. Establishing efficient conduction paths is somewhat complicated. The three structural features of HNAs offer variety for the combination of bandgap and band location. Table 1 selects successful binary designs from the literatures that highlight corresponding findings with the charge transfer across the interface of different constituents.

Table 1. Summary of selected binary heterogenerous nanostructure arrays with 'Function-Function' mode

\begin{tabular}{|c|c|c|c|c|c|c|}
\hline \multirow{2}{*}{ Heterostructure } & \multirow{2}{*}{$\begin{array}{l}\text { Morpholo } \\
\text { gy of unit }\end{array}$} & \multirow{2}{*}{$\begin{array}{c}\text { Bandgap } \\
\text { I (eV) }\end{array}$} & \multirow{2}{*}{$\begin{array}{c}\text { Bandgap } \\
\text { II }(\mathrm{eV})\end{array}$} & \multicolumn{2}{|c|}{$\begin{array}{l}\text { Suggested charge } \\
\text { transfer across the } \\
\text { interface }\end{array}$} & \multirow[t]{2}{*}{ Ref. } \\
\hline & & & & $\begin{array}{c}\text { Electro } \\
\mathrm{n}\end{array}$ & Hole & \\
\hline $\mathrm{Si}-\mathrm{TiO}_{2}$ & $\begin{array}{l}\text { Core-shell } \\
\text { nanowire }\end{array}$ & 1.12 & 3 & l & I & [79] \\
\hline p-Si-TiO 2 & $\begin{array}{l}\text { Core-shell } \\
\text { nanowire }\end{array}$ & 1.12 & 3 & $\mathrm{Si}-\mathrm{TiO}_{2}$ & $\mathrm{TiO}_{2}-\mathrm{Si}$ & [79] \\
\hline $\mathrm{Si}-\mathrm{TiO}_{2}$ & $\begin{array}{l}\text { Branched } \\
\text { nanowire }\end{array}$ & 1.1 & 3 & 1 & 1 & {$[80]$} \\
\hline $\mathrm{Si}-\mathrm{Fe}_{2} \mathrm{O}_{3}$ & $\begin{array}{l}\text { Core-shell } \\
\text { nanowire }\end{array}$ & 1.1 & 2.1 & I & 1 & $\begin{array}{l}{[75,} \\
81]\end{array}$ \\
\hline $\mathrm{TiO}_{2}-\mathrm{SnO}_{2}$ & Core-shell & 2.82 & 3.2 & $\mathrm{TiO}_{2}-\mathrm{S}$ & $\mathrm{SnO}_{2}-\mathrm{Ti}$ & [82] \\
\hline
\end{tabular}




\begin{tabular}{|c|c|c|c|c|c|c|}
\hline & $3 \mathrm{DOM}$ & & & $\mathrm{nO}_{2}$ & $\mathrm{O}_{2}$ & \\
\hline $\mathrm{ZnO}-\mathrm{TiO}_{2}$ & $\begin{array}{l}\text { Dot on } \\
\text { nanowire }\end{array}$ & 3.3 & 3.2 & $\begin{array}{l}\mathrm{TiO}_{2}-\mathrm{Z} \\
\mathrm{nO}\end{array}$ & ${ }_{2} \mathrm{ZnO}-\mathrm{TiO}$ & {$[83]$} \\
\hline $\mathrm{WO}_{3}-\mathrm{TiO}_{2}$ & $\begin{array}{l}\text { Core-shell } \\
\text { nanorod }\end{array}$ & 2.8 & 3.2 & $\begin{array}{l}\mathrm{TiO}_{2-} \\
\mathrm{WO}_{3}\end{array}$ & $\begin{array}{l}\mathrm{WO}_{3-} \\
\mathrm{TiO}_{2}\end{array}$ & {$[84]$} \\
\hline $\mathrm{TiO}_{2}-\mathrm{g}-\mathrm{C}_{3} \mathrm{~N}_{4}$ & $\begin{array}{l}\text { Core-shell } \\
\text { nanotube }\end{array}$ & 3.2 & 2.7 & $\begin{array}{l}\text { g- } \mathrm{C}_{3} \mathrm{~N}_{4}- \\
\mathrm{TiO}_{2}\end{array}$ & $\begin{array}{l}\mathrm{TiO}_{2}-\mathrm{g}-\mathrm{C} \\
{ }_{3} \mathrm{~N}_{4}\end{array}$ & {$[85]$} \\
\hline $\mathrm{TiO}_{2}-\mathrm{CdS}$ & $\begin{array}{l}\text { Core-shell } \\
\text { nanorod }\end{array}$ & 3.2 & 2.39 & $\begin{array}{l}\mathrm{CdS}-\mathrm{Ti} \\
\mathrm{O}_{2}\end{array}$ & $\begin{array}{l}\mathrm{TiO}_{2}-\mathrm{Cd} \\
\mathrm{S}\end{array}$ & {$[86]$} \\
\hline $\mathrm{TiO}_{2}-\mathrm{CdSe}$ & $\begin{array}{l}\text { Core-shell } \\
\text { nanorod }\end{array}$ & 3.2 & 1.73 & $\begin{array}{l}\mathrm{CdSe}-\mathrm{T} \\
\mathrm{iO}_{2}\end{array}$ & $\begin{array}{l}\mathrm{TiO}_{2}-\mathrm{Cd} \\
\mathrm{Se}\end{array}$ & {$[86]$} \\
\hline $\mathrm{TiO}_{2}-\mathrm{CdSeS}$ & $\begin{array}{l}\text { Core-shell } \\
\text { nanorod }\end{array}$ & 3.2 & 2.07 & $\begin{array}{l}\text { CdSeS- } \\
\mathrm{TiO}_{2}\end{array}$ & $\begin{array}{l}\mathrm{TiO}_{2}-\mathrm{Cd} \\
\mathrm{SeS}\end{array}$ & {$[86]$} \\
\hline $\mathrm{TiO}_{2}-\mathrm{MoS}_{2}$ & $\begin{array}{l}\text { Nanosheet } \\
\text { on } \\
\text { nanorod }\end{array}$ & 3.11 & 1.71 & l & $\begin{array}{l}\mathrm{TiO}_{2}-\mathrm{Mo} \\
\mathrm{S}_{2}\end{array}$ & {$[87]$} \\
\hline $\mathrm{TiO}_{2}-\mathrm{ZnFe}_{2} \mathrm{~S}_{4}$ & $\begin{array}{l}\text { Nanosheet } \\
\text { on } \\
\text { nanorod }\end{array}$ & 3 & 2.91 & $\begin{array}{l}\mathrm{ZnFe} 2 \mathrm{~S} \\
4-\mathrm{TiO}_{2}\end{array}$ & $\begin{array}{l}\mathrm{TiO}_{2}-\mathrm{Zn} \\
\mathrm{Fe}_{2} \mathrm{~S}_{4}\end{array}$ & {$[88]$} \\
\hline $\mathrm{V}: \mathrm{TiO}_{2}-\mathrm{In}_{2} \mathrm{~S}_{3}$ & $\begin{array}{l}\text { Core-shell } \\
\text { nanorod }\end{array}$ & 2.96 & 2.3 & $\begin{array}{l}\mathrm{In}_{2} \mathrm{~S}_{3-} \\
\mathrm{V}: \mathrm{TiO}_{2}\end{array}$ & $\begin{array}{l}\mathrm{V}: \mathrm{TiO}_{2}-\mathrm{I} \\
\mathrm{n}_{2} \mathrm{~S}_{3}\end{array}$ & {$[88]$} \\
\hline $\mathrm{TiO}_{2}-\mathrm{BiVO}_{4}$ & $\begin{array}{l}\text { Nanodot } \\
\text { on 3DOM }\end{array}$ & 3.2 & 2.4 & $\begin{array}{l}\mathrm{BiVO}_{4-} \\
\mathrm{TiO}_{2}\end{array}$ & $\begin{array}{l}\mathrm{TiO}_{2}-\mathrm{Bi} \\
\mathrm{VO}_{4}\end{array}$ & [89] \\
\hline $\mathrm{TiO}_{2}-\mathrm{BiFeO}_{3}$ & $\begin{array}{l}\text { Nanodot } \\
\text { on } \\
\text { nanowire }\end{array}$ & 3.16 & 2.12 & $\begin{array}{l}\mathrm{BiFeO}_{3} \\
-\mathrm{TiO}_{2}\end{array}$ & $\begin{array}{l}\mathrm{TiO}_{2}-\mathrm{BiF} \\
\mathrm{eO}_{3}\end{array}$ & {$[90]$} \\
\hline $\begin{array}{l}\mathrm{Co}: \mathrm{Fe}_{2} \mathrm{O}_{3}-\mathrm{MgF} \\
\mathrm{e}_{2} \mathrm{O}_{4}\end{array}$ & $\begin{array}{l}\text { Core-shell } \\
\text { nanorod }\end{array}$ & 2.1 & 2.4 & $\begin{array}{l}\mathrm{MgFe}_{2} \\
\mathrm{O}_{4}-\mathrm{Co}: \\
\mathrm{Fe}_{2} \mathrm{O}_{3}\end{array}$ & $\begin{array}{l}\mathrm{Co}: \mathrm{Fe}_{2} \mathrm{O} \\
3-\mathrm{MgFe}_{2} \\
\mathrm{O}_{4}\end{array}$ & {$[35]$} \\
\hline $\mathrm{SnO}_{2}-\mathrm{WO}_{3}$ & $\begin{array}{l}\text { Core-shell } \\
\text { 3DOM }\end{array}$ & 3.8 & $2.6-2.8$ & $\begin{array}{l}\mathrm{WO}_{3}-\mathrm{S} \\
\mathrm{nO}_{2}\end{array}$ & $\begin{array}{l}\mathrm{SnO}_{2-} \mathrm{W} \\
\mathrm{O}_{3}\end{array}$ & [91] \\
\hline $\mathrm{ZnO}-\mathrm{ZnO} / \mathrm{ZnS}$ & Core-shell & 3.19 & 3.08 & $\begin{array}{l}\mathrm{ZnO} / \mathrm{Zn} \\
\mathrm{S} \text { solid }\end{array}$ & $\begin{array}{l}\mathrm{ZnO}-\mathrm{Zn} \\
\mathrm{O} / \mathrm{ZnS}\end{array}$ & {$[92]$} \\
\hline
\end{tabular}




\begin{tabular}{l|l|l|l|l|l|l}
\hline solid solution & nanorod & & & $\begin{array}{l}\text { solution } \\
-\mathrm{ZnO}\end{array}$ & $\begin{array}{l}\text { solid } \\
\text { solution }\end{array}$ & \\
\hline $\mathrm{WO}_{3}-\mathrm{BiVO}_{4}$ & $\begin{array}{l}\text { Core-shell } \\
\text { nanorod }\end{array}$ & 2.6 & 2.4 & $\begin{array}{l}\mathrm{BiVO}_{4}- \\
\mathrm{WO}_{3}\end{array}$ & $\begin{array}{l}\mathrm{WO}_{3}-\mathrm{Bi} \\
\mathrm{VO}_{4}\end{array}$ & $\begin{array}{l}{[93,} \\
94]\end{array}$ \\
\hline $\mathrm{BiVO}_{4}-\mathrm{FeVO}_{4}$ & $\begin{array}{l}\text { Core-shell } \\
3 \mathrm{DOM}\end{array}$ & 2.4 & 2.06 & $\begin{array}{l}\mathrm{FeVO}_{4}- \\
\mathrm{BiVO}_{4}\end{array}$ & $\begin{array}{l}\mathrm{BiVO}_{4}-\mathrm{F} \\
\mathrm{eVO}_{4}\end{array}$ & {$[95]$} \\
\hline $\mathrm{GaN}-\mathrm{InGaN}$ & $\begin{array}{l}\text { Core-shell } \\
\text { nanorod }\end{array}$ & 3.4 & 2.36 & $/$ & $\begin{array}{l}\mathrm{GaN}-\mathrm{InG} \\
\mathrm{aN}\end{array}$ & {$[96]$} \\
\hline
\end{tabular}

\subsection{Function-Assistance}

For 'Function-Assistance' mode, one functional constituent undertake the main tasks of solar water splitting. Meanwhile, related properties can be adjusted by the other constituents for better adapting the electrochemical processes, including light harvesting, charge migration, charge separation, surface kinetic, and stability, etc.

(1) Enhanced light harvesting

Employing different constituents to assist the absorption of functional constituent is another way to improve the light harvesting. One wildly-used approach is the introduction of upconversion materials to suppress the transmission of sub-bandgap photons by converting two sub-bandgap photons into one above-bandgap photon [97]. Figure 2 shows a typical example about $\mathrm{TiO}_{2}$ 3DOM/NaYF $4: \mathrm{Yb}^{3+} / \mathrm{Er}^{3+} @ \mathrm{NaYF}_{4}$ core-shell nanoparticle/CdSe shell. Upconversion constituent will not directly join in the water splitting reactions but allows for enhanced near-infrared light harvesting and solar conversion efficiency under infrared light (980 $\mathrm{nm}$ as an example in Figure 2c) [97]. Other upconversion materials like $\beta-\mathrm{NaYF}_{4}: \mathrm{Yb}^{3+} / \mathrm{Tm}^{3+}$ [98], $\mathrm{Er}_{2} \mathrm{O}_{3}$ [99], carbon quantum dot [100], etc. have also used to enhance the range of light absorption and further improve the solar conversion ability. 

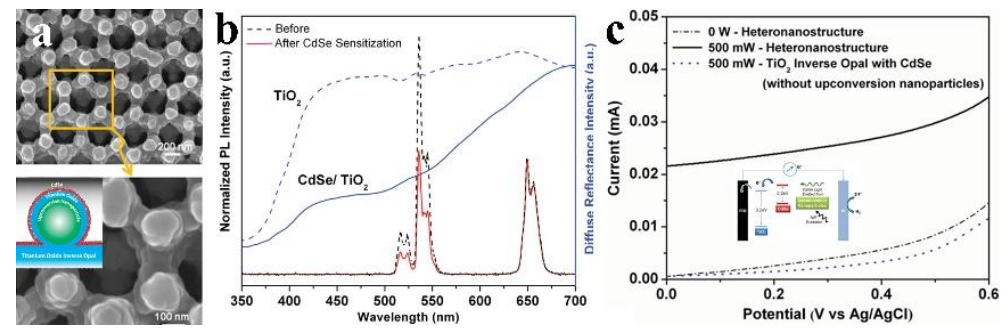

Figure 2. Typical examples of heterogeneous nanostructure arrays with 'Function-Assistance' working mode for enhanced light harvesting by means of upconversion materials. (a) Top view and a magnified image of $\mathrm{TiO}_{2}$ 3DOM/NaYF $4: \mathrm{Yb}^{3+} / \mathrm{Er}^{3+} @ \mathrm{NaYF}_{4}$ core-shell nanoparticle/CdSe shell with schematic illustration inset. (b) Upconversion emission spectra of upconversion constituents and diffuse reflectance measurement before and after the coating of CdSe quantum dots. (c) Photocurrent density-potential curves under $980 \mathrm{~nm}$ laser diode excitation powered at $500 \mathrm{~mW}$. Reproduced with permission. [97] Copyright 2013, Wiley-VCH Verlag GmbH \& Co. KGaA.

Besides, surface plasmon resonance (SPR) effect originated from the plasmonic constituents has also been proved as an efficient approach with more diversity and controllibility to enhance light absorption [101-103]. Plasmonic constituents always consisted of $\mathrm{Au}, \mathrm{Ag}, \mathrm{Bi}, \mathrm{Al}, \mathrm{Cu}$ and other metals that will not directly take part in the reactions of water splitting, but contribute on resonant photon-induced collective oscillation of valence electrons of functional constituents [104, 105], First, plasmonic constituents can fold incident light into functional constituents through directional scattering and intense electric-field-aided light absorption around the plasmonic constituents [106]. Second, with the help of plasmonic constituents, it is possible to sensitize the functional constituents to below bandgap light and inject hot electrons/hot holes into functional constituents [107]. Third, reflection losses from the electrode surfaces can be decreased to a nearly negligible level because of the high refractive index of plasmonic metal [107]. Forth, the response location of SPR is easy to be adjusted by changing the size, dimensionality, morphology and alignment of plasmonic constituents. 
Nowadays, various plasmonic metallic nanostructures have been designed as cores for coating shells of functional constituents, such as nanodot array [108, 109], nanohole array [110], nanocone array [111], nanorod array [112, 113], nanowire array [114] and so on. Xu et al. recently develop a novel CdSe/Au pillar/truncated-pyramid (PTP) array as a plasmonic coupler shown in Figure 3a [106]. According to the absorption spectrum based on finite difference time domain simulations (Figure 3b), SPR modes contribute enhancement in the longer wavelength range $(>450 \mathrm{~nm})$, and photonic modes contribute mainly in the shorter wavelength range $(<450 \mathrm{~nm})$. The photocurrent density reaches $3.5 \mathrm{~mA} \mathrm{~cm}^{-2}$, which is four times than that of the planar $\mathrm{CdS} / \mathrm{Au}$ (Figure 3c). In addition, plasmonic constituents can disperse on the surface of functional constituents $[115,116]$ or be embedded into functional constituents [117]. For example, $20 \mathrm{~nm}$ Au nanoparticles has been decorated on the surface of $\mathrm{Mo}: \mathrm{BiVO}_{4}$ 3DOM architectures as shown in Figure 3d. Synergistic effects of photonic bands and plasmonic absorption are observed to obviously enhance light absorption (Figure 3e) and photocurrent density of $3.1 \pm 0.1 \mathrm{~mA} \mathrm{~cm}^{-2}$ at $1.23 \mathrm{~V}$ vs RHE (Figure 3f) [118]. Another example is $\mathrm{ZnS}-\mathrm{Ag}-\mathrm{CdS}-\mathrm{Au}-\mathrm{CdSe}$ nanobamboo arrays in Figure 3g-3i [119]. The SPR effects of Ag and Au constituents allow the possibilities of large scattering cross-section, resulting in increased effective optical length inside the nearby functional constituents and thereby enhanced their light absorption. Meanwhile, because of the absorption spectrum overlap between plasmonic constituents and functional constituents, the energy in localized plasmonic oscillations transfers from $\mathrm{Ag}$ and $\mathrm{Au}$ constituents to their neighboring constituents. Hence, more electron-hole pairs will be generated on the basis of improved light absorption from above two factors and produce high photocurrent. 

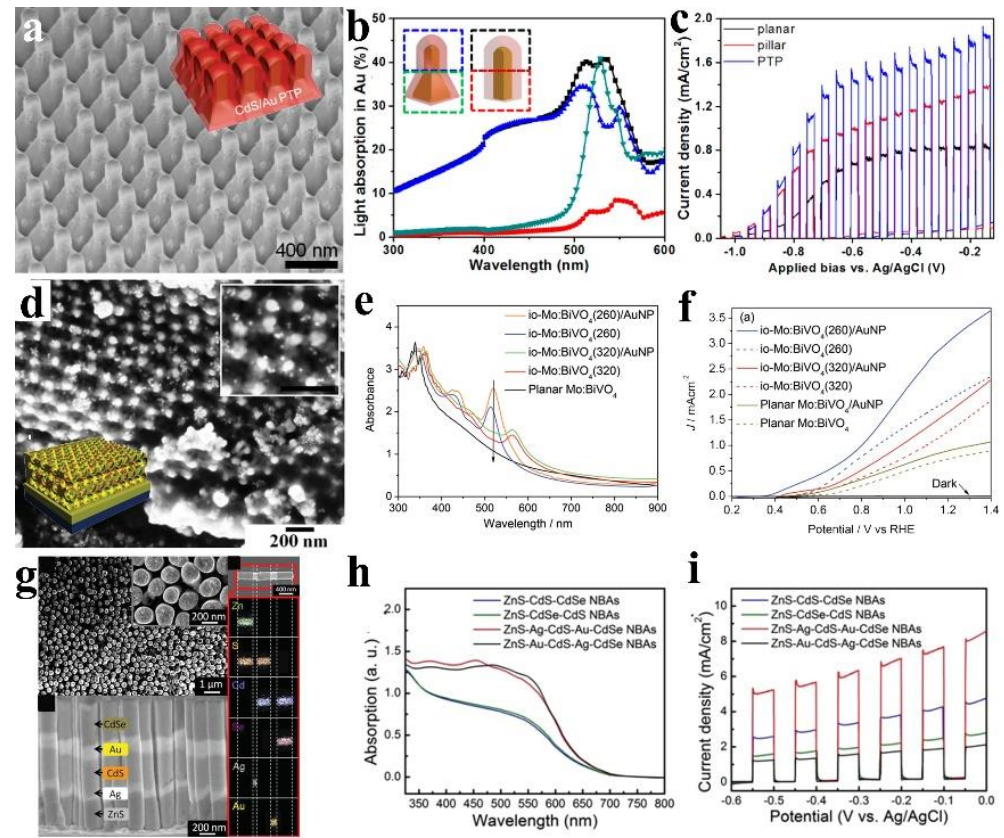

Figure 3. Typical examples of heterogeneous nanostructure arrays with 'Function-Assistance' working mode for enhanced light harvesting by means of surface plasmon resonance (SPR) effect. (a-c) SEM image, finite difference time domain (FDTD) simulation of absorption curves and chopped photocurrent density-potential curves of CdS/Au PTP array. Reproduced with permission. [106] Copyright 2017, American Chemical Society. (d-f) Top view, absorption spectra and photocurrent density-potential curves of $\mathrm{Au} / \mathrm{Mo}: \mathrm{BiVO}_{4}$ three-dimensionally ordered mesoporous (3DOM) array. Reproduced with permission. [118] Copyright 2014, Wiley-VCH Verlag GmbH \& Co. KGaA. (g) Top view and side views of ZnS-Ag-CdS-Au-CdSe nanobamboo array with EDS elemental mapping analysis of a single unit. (h,i) absorption spectra and photocurrent density-potential curves of nanobamboo arrays. Reproduced with permission. [119] Copyright 2015, Wiley-VCH Verlag GmbH \& Co. KGaA.

(2) Improved charge separation and migration

Inspired by nature, scientists always employ semiconductors as functional constituents to separate photo-generated charges. However, most semiconductors suffer from poor charge migration, and thus photo-generated holes and electrons will 
be recombined in a fast rate to reduce the achievable photo-conversion efficiencies. Engineering of size, dimensionality and morphology is a potential approach to address this issue, but still limited by a single material to carry out the functions of both light absorption and charge migration [14]. The idea of optimizing surface/interface state with extra electric field or introducing highly conductive constituents along charge collection paths holds great promise to assist charge separation and migration and avoid unfavorable charge recombination of functional constituents. SPR is a typical solution to introduce local electric field near the interface. As shown in $\mathrm{ZnS}-\mathrm{Ag}-\mathrm{CdS}-\mathrm{Au}-\mathrm{CdSe}$ nanobamboo arrays in Figure 3g, enhanced electromagnetic fields originated from the strong SPR of Ag and Au constituents may also help for the spatial charge separation in contiguous functional constituents, thereby, effectively assisting the charge separation [120]. The SPR-induced local electric-field intensity can be adjusted by controlling the size dimensionality and morphology of SPR constituents. Similar enhancement can also be observed in other functional constituents assisted by SPR assistance constituents, such as multi-segmented $\mathrm{CdS}-\mathrm{Au}$ nanorod array [121], a sandwich-structured $\mathrm{CdS}-\mathrm{Au}-\mathrm{TiO}_{2}$ nanorod array [117], Au-N-doped $\mathrm{TiO}_{2}$ bowl nanoarray [115], etc. Another solution to introduce local electric field is employing ferroelectric polarization to engineer the interfacial electronic band structure. $\mathrm{SrTiO}_{3}$ is a typical material with strong ferroelectrics. After coating $\mathrm{SrTiO}_{3}$ onto $\mathrm{TiO}_{2}$ nanowire array, the resulting shell-core architecture (Figure 4a) exhibits improved performance of solar water splitting, in particular after positive poling (Figure 4b). It indicates that the existence of positive ferroelectric polarization of $\mathrm{SrTiO}_{3}$ can induce upward band bending of $\mathrm{TiO}_{2}$ as diagramed in Figure 4c. Such amplified width and amplitude of interfacial band bending can effectively force charge separation and migration, so that reduced charge recombination contributes to remarkable improvement of solar water splitting [122]. These results highlight the vital influences of controllable surface/interface state on optimizing charge separation and migration in an integrated system. 
Compared with adjustment of surface/interface state, introducing conductive constituents is a straight-forward and effective solution to drive charge migration and alleviate charge recombination. Exploiting multi-dimensional current collectors can build a direct and short path for charge migration and serve as the supporters to keep the superiorities of functional constituents under nanoscale level. Numerous highly conductive materials, including $\mathrm{TiSi}_{2}$, graphene, fluorine-doped tin oxide (FTO), tin-doped indium oxide (ITO), $\mathrm{Al}$ doped $\mathrm{ZnO}(\mathrm{AZO})$, etc. have been fabricated into different nanostructure arrays as current collectors for improved solar water splitting. $\mathrm{TiO}_{2}$ is an effective active material for solar water splitting but suffers from low electron mobility $\left(1 \mathrm{~cm} 2 \mathrm{~V}^{-1} \mathrm{~s}^{-1}\right)$ and hole diffusion length $(10-100 \mathrm{~nm})$. To address this issue, $\mathrm{Mi}$ et al. have developed a well-ordered $\mathrm{AZO} / \mathrm{TiO}_{2}$ core/shell nanocone array based on AAO template (Figure 4d). The presence of AZO provides a conductive path and facilitate the electrons separation and transport simultaneously in short horizontal direction instead of long vertical direction, leading to three times enhancement of that from planar $\mathrm{AZO} / \mathrm{TiO}_{2}$ as shown in Figure 4e [123]. Lin et al. design a $\mathrm{TiO}_{2} / \mathrm{TiSi}_{2}$ core/shell nanonet array to ease this issue. Highly conductive $\mathrm{TiSi}_{2}$ cores [124] can form a dedicated charge transporter for other materials with poor conductivity, such as $\mathrm{TiO}_{2}, \mathrm{Fe}_{2} \mathrm{O}_{3}$. Shown in Figure $4 \mathrm{f}$ is $\mathrm{TiSi}_{2}$ nanonet directly interfaced with thin $\mathrm{Fe}_{2} \mathrm{O}_{3}$ shell. The distance from charge generation to a location where charge can be transported by $\mathrm{TiSi}_{2}$ is short, allowing for enhanced charge separation/collection and high photocurrent density (Figure 4g) [125].
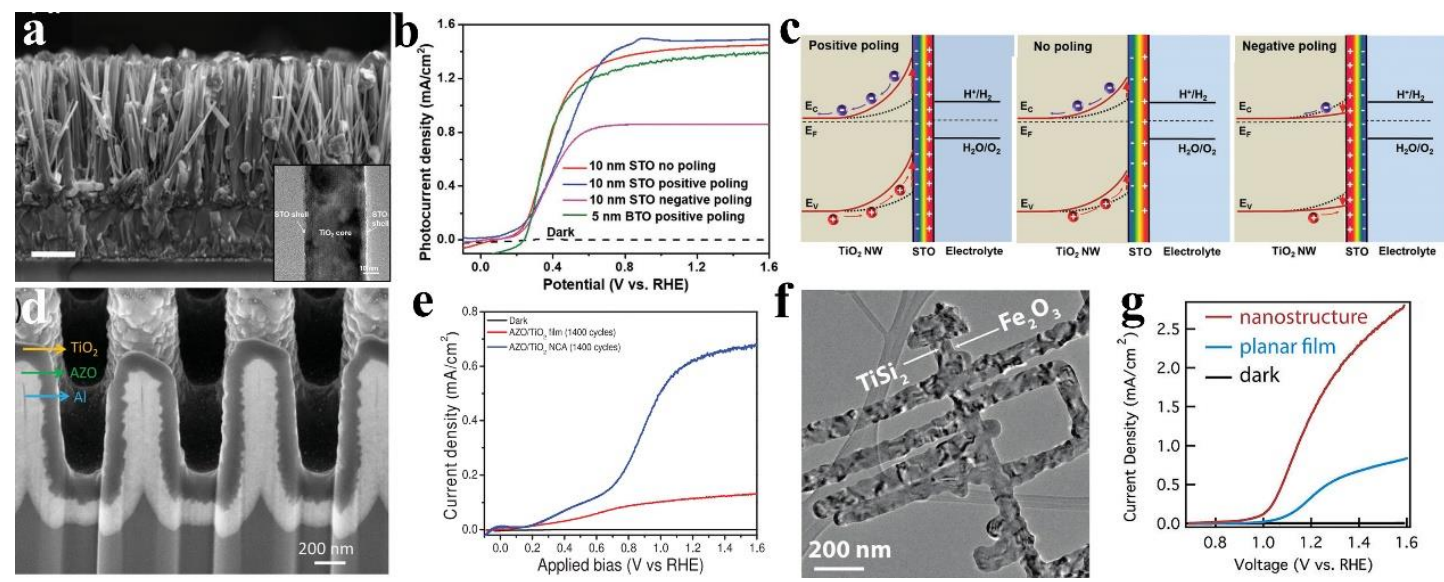
Figure 4. Typical examples of heterogeneous nanostructure arrays with 'Function-Assistance' working mode for improved charge separation and migration by means of local electric field at surface (a-c) and multi-dimensional current collectors (d-g). (a) Top-view of $\mathrm{TiO}_{2} / \mathrm{SrTiO}_{3}$ core-shell nanowire array with TEM image of individual unit, (b) Photocurrent density-potential curves of $\mathrm{TiO}_{2} / 10 \mathrm{~nm}$ $\mathrm{SrTiO}_{3}$ core-shell nanowire array, (c) Schematic electronic band diagram of the $\mathrm{TiO}_{2}-$ $\mathrm{SrTiO}_{3}$ interface with positive poling, no poling and negative poling conditions. Reproduced with permission. [122] Copyright 2017, Wiley-VCH Verlag GmbH \& Co. KGaA. (d,e) Cross-section view and photocurrent density-potential curves of Al doped $\mathrm{ZnO}(\mathrm{AZO}) / \mathrm{TiO}_{2}$ nanocone array. Reproduced with permission. [123] Copyright 2017, Wiley-VCH Verlag GmbH \& Co. KGaA. (f,g) Transmission electron microscope (TEM) images and photocurrent density-potential curves of $\mathrm{TiSi}_{2} / \mathrm{Fe} 2 \mathrm{O}_{3}$ nanonet array. Reproduced with permission. [125] Copyright 2011, American Chemical Society.

\section{(3) Fast surface reaction}

Considering that water oxidation and proton reduction at surface is somewhat complex processes due to four- and two-electron/proton nature, rates of surface reactions are thus regarded as one of the main limitations in solar water splitting. Deep understandings of corresponding thermodynamics and kinetics are important for improvement of solar water splitting. One solution to facilitate surface reaction is the integration of surface-attached electrocatalysts as assistance constituents. As such, first, the photo-generated charges can be transferred to active sites with lower activation energy for surface water splitting, such as Pt for hydrogen evolution. Second, surface charge recombination will be suppressed, associating with facile charge separation. Cummings et al. pioneered kinetic and mechanistic researches on the "catalytic" effect by surface treatment of $\mathrm{Co}(\mathrm{II})$ on $\mathrm{Fe}_{2} \mathrm{O}_{3}$ [126], and proved that the role of electrocatalysts is the suppression of surface recombination. Based on the two advantages, lots of highly active electrocatalysts, including $\mathrm{Pt}, \mathrm{MoS}_{2}, \mathrm{NiFeO}_{\mathrm{x}}$, 
amorphous cobalt phosphate $(\mathrm{Co}-\mathrm{Pi}), \mathrm{FeOOH}, \mathrm{NiOOH}, \mathrm{Co}(\mathrm{OH})_{2}$, etc. have been modified on different functional constituents with array morphologies in pursuit of effective surface reactions. $\mathrm{BiVO}_{4} / \mathrm{Co}-\mathrm{Pi}$ core-shell nanowall array (Figure $5 \mathrm{a}-\mathrm{c}$ ) is a typical instance. The presence of Co-Pi can abate surface recombination and achieve $\sim 100 \%$ efficiency of interfacial charge transfer, permitting a remarkable cathodic shift of onset potential to $\sim 320 \mathrm{mV}$ was achieved [127]. Third, the direct contact between electrocatalytic constituents and functional constituents enable the creation of new interfaces, which are of significance for the capabilities of charge separation and transfer. $\mathrm{Ta}_{3} \mathrm{~N}_{5} / \mathrm{Co}(\mathrm{OH})_{2}$ core/shell nanotube array is a representative example as shown in Figure 5d. After photoelectrochemical (PEC) treatment, the formation of Ta-O-Co bonding (Figure 5d) between $\mathrm{Ta}_{3} \mathrm{~N}_{5}$ and $\mathrm{Co}(\mathrm{OH})_{2}$ can suppress Fermi-level pinning when compared with normal Ta-O-Ta bonds at the pure $\mathrm{Ta}_{3} \mathrm{~N}_{5} /$ electrolyte interface. Such new photo-induced interface is benefit for not only charge separation, but also accelerating charge transfer to desired reactions, leading to better capabilities of solar water splitting (Figure 5e) [128].

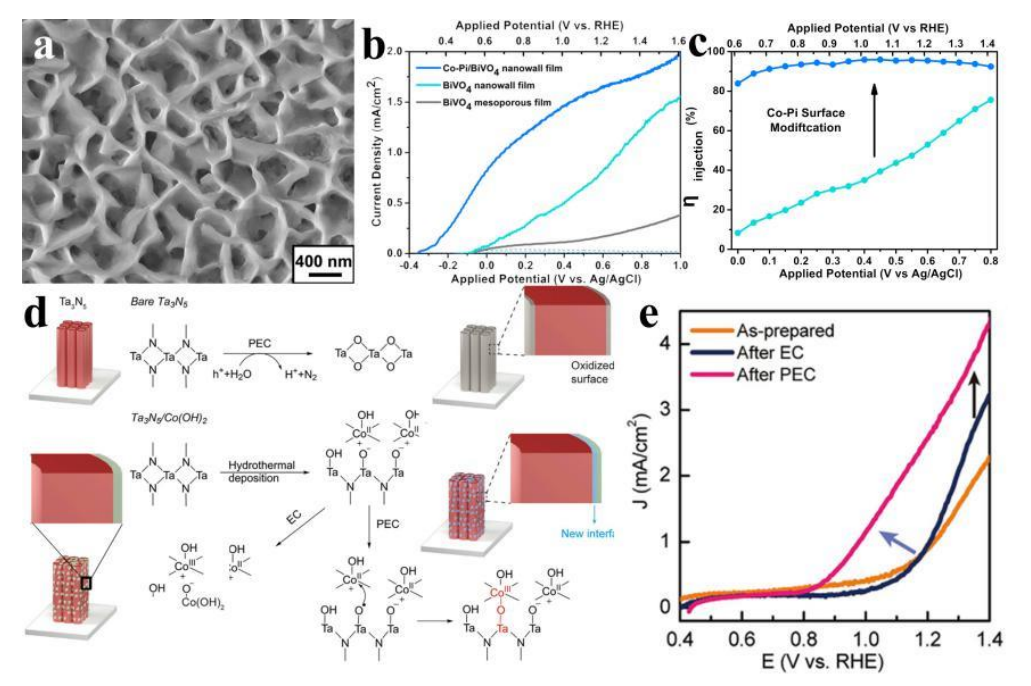

Figure 5. Typical examples of heterogeneous nanostructure arrays with 'Function-Assistance' working mode for fast surface reaction by means of surface-attached electrocatalysts (a-c) and new photo-induced interface (d,e). (a-c) Top-view of $\mathrm{Co}-\mathrm{Pi} / \mathrm{BiVO}_{4}$ nanowall array, (b) Photocurrent density-potential curves 
and (c) interfacial charge transfer efficiency of $\mathrm{Co}-\mathrm{Pi} / \mathrm{BiVO}_{4}$ nanowall array under the illumination of $100 \mathrm{~mW} \mathrm{~cm}{ }^{-2}$. Reproduced with permission. [127] Copyright 2012, Wiley-VCH Verlag GmbH \& Co. KGaA. (d) Proposed mechanism for the formation of the Ta-O-Co bond in $\mathrm{Ta}_{3} \mathrm{~N}_{5}$ nanowire array under photo-electrochemical (PEC) conditions, (e) Photocurrent density-potential curves after electrochemical (EC) and PEC treatment in $1 \mathrm{M} \mathrm{NaOH}$. EC treatment, dark electrolysis at $2 \mathrm{~V}$ for $10 \mathrm{~min}$; PEC treatment, photo-electrolysis at $1.23 \mathrm{~V}$ for $10 \mathrm{~min}$. Reproduced with permission. [128] Copyright 2017, Cell Press.

Another solution is the application of ultrathin coatings by means of surface electron tunneling, dangling bond passivation or electronic structure adjustment. These configurations hold the potential to alleviate restrictions associated with incompatible band alignments. $\mathrm{Fe}_{2} \mathrm{O}_{3}$ is a functional constituent that has attracted great attention on ultrathin surface coating because of its poor water oxidation kinetics [15]. With the help of ultrathin $\mathrm{Al}_{2} \mathrm{O}_{3}$ coating, $\mathrm{Al}_{2} \mathrm{O}_{3} / \mathrm{Fe}_{2} \mathrm{O}_{3}$ cauliflower array shows cathodic shift of onset potential $(\sim 100 \mathrm{mV})$ and a photocurrent density of $0.42 \mathrm{~mA}$ $\mathrm{cm}^{-2}$ at $0.9 \mathrm{~V}$ vs. RHE after modification with $\mathrm{Co}^{2+}$ catalyst. Analysis from electrochemical impedance and photoluminescence spectroscopy evidences that the remarkable results originate from the passivation of surface states and suppression of surface recombination rather than catalytic effects [129].

(4) Long-term stability

Stability is one of the most important parameter for solar water splitting. In many cases, functional constituents suffer from photocorrosion because of accumulated energetic intermediates, such as high concentration of $\mathrm{OH}-$, self-reduction/oxidation by photo-generated charges. The preventive strategy is to employ an assisted surface protective layer to isolate the functional constituents from reactive species, sometimes associating with additional welfare like reduced surface charge recombination. $\mathrm{Cu}_{2} \mathrm{O}$ is a promising p-type candidate for hydrogen evolution with low stability in electrolyte owning to the redox potential of $\mathrm{Cu}_{2} \mathrm{O}$ lies within its bandgap. Paracchino 
et al. deposited n-type $\mathrm{Al}$ doped $\mathrm{ZnO}(\mathrm{AZO})$ and $\mathrm{TiO}_{2}$ hybrid films on the $\mathrm{Cu}_{2} \mathrm{O}$ array (Figure 6a). Photo-generated electrons can be forced by the band offset to transfer from $\mathrm{Cu}_{2} \mathrm{O}$ through the protective layer to the electrolyte and join in the water reduction reactions with $\sim 100 \%$ Faradaic efficiency. Compared with pure $\mathrm{Cu}_{2} \mathrm{O}$, the protected $\mathrm{Cu}_{2} \mathrm{O}$ array can remain active after 1 hour of testing without formation of metallic $\mathrm{Cu}$ particles (Figure $6 \mathrm{~b}-\mathrm{c}$ ) [130]. $\mathrm{Ta}_{3} \mathrm{~N}_{5}$ nanowire array is also benefit from complete separation from $\mathrm{H}_{2} \mathrm{O}$ and reactive $\mathrm{O}$ species by surface passivation layer of amorphous oxynitridie $(<3 \mathrm{~nm})$ and $\mathrm{MgO}$ by atomic layer deposition (ALD). Such heterogeneous array can remain stable for $30 \mathrm{~min}$ in $1 \mathrm{M} \mathrm{NaOH}$ at $1.23 \mathrm{~V}$ vs RHE [131]. Similar strategy is also suitable for $\mathrm{Si}$. 8-nm $\mathrm{TiO}_{2}$ layer by ALD extends the operational lifetime of black silicon (b-Si) from less than 0.5 hour to 4 hours. (Figure 6d-f) [132].
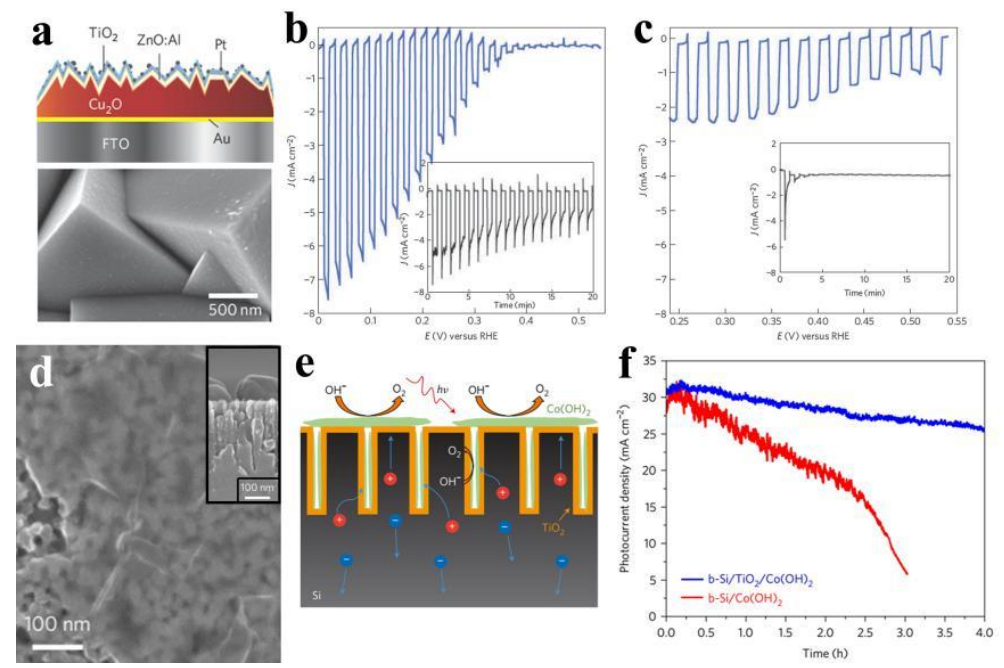

Figure 6. Typical examples of heterogeneous nanostructure arrays with 'Function-Assistance' working mode for long-term stability. (a) Schematic representation and SEM image of surface-protected $\mathrm{Cu}_{2} \mathrm{O}$ electrode, (b,c) The photoelectrochemical response for the surface-protected $\mathrm{Cu}_{2} \mathrm{O}(\mathrm{b})$ and bare $\mathrm{Cu}_{2} \mathrm{O}$ (c) under chopped AM 1.5 light illumination. The insets show the photocurrent transients at $0 \mathrm{~V}$ vs RHE in chopped light illumination with $\mathrm{N}_{2}$ purging. Reproduced with permission. [130] Copyright 2011, Springer Nature. (d) Top-view and cross-sectional (inset) $\mathrm{SEM}$ images of $\mathrm{b}-\mathrm{Si} / \mathrm{TiO}_{2} / \mathrm{Co}(\mathrm{OH})_{2}$, (e) Schematic illustration of 
b-Si $/ \mathrm{TiO}_{2} / \mathrm{Co}(\mathrm{OH})_{2}$, (f) Photocurrent transients at $1.65 \mathrm{~V}$ vs RHE under 1 sun illumination. Reproduced with permission. [132] Copyright 2017, Springer Nature.

\subsection{Single-unit device}

Both the two as-mentioned modes of HNAs aim at optimizing the electrochemical processes of either oxygen evolution or hydrogen production. With the development of advanced fabrication techniques, instruments, device engineering and understanding of electrochemical mechanisms, the ultimate goal in the field of solar water splitting is realizing unassisted artificial photosysthsis that uses solar energy to produce chemicals with a high efficiency and long-term operational stability. Inspired by individual natural photosynthetic organisms, one can image a stable and scalable design [133] to create a nanostructure array, of which each unit is a photoelectrochemically active device with autonomous functions of oxygen evolution and hydrogen production. Each single-unit device always consists of efficient semiconductor constituents as light absorbers in contact with oxidation and reduction electrocatalysts. The photo-generated electrons and holes can be separated by internal electric potential, and then migrate to suitable reactive sites. Such large numbers of single-unit devices can be assembled into a macroscopical array, so that huge energy output can be highly expected. Several HNAs with 'single-unit device' mode have been successfully achieved with four typical kinds. (1) The first design comes from the understanding of SPR. As exhibited in Figure 7a and inset of 7c, Au nanorod array based on anodic aluminium oxide (AAO) templates is uniformly coated with a thin layer of Pt-decorated $\mathrm{TiO}_{2}$ on the top and cobalt-based oxygen evolution catalyst (Co-OEC) on the bottom sides. The hot electrons produced by surface plasmons can inject into the conduction band of $\mathrm{TiO}_{2}$ (Figure $7 \mathrm{~b}$ ) and then migrate to the $\mathrm{Pt}$ nanoparticles to join in the water-reduction reactions for hydrogen generation, permitting $5 \times 10^{13} \mathrm{H}_{2}$ molecules $\mathrm{cm}^{-2} \mathrm{~s}^{-1}$ under 1 sun illumination with long-term operational stability (Figure 7c). The holes in the Au nanorod array migrate to the contact zone of $\mathrm{Au} / \mathrm{Co}-\mathrm{OEC}$ for oxygen generation. Although the reported 
solar-to-hydrogen efficiency is only $0.1 \%$, that is too low for practical usage. But straightforward structural optimization is expected for significant efficiency improvements [134]. (2) The separation of photo-generated charges can be completed by means of photovoltaic part. A multisegmented poly(3,4-ethylenedioxythiophene) $[\mathrm{PEDOT}] / \mathrm{CdSe} / \mathrm{Ni} / \mathrm{Au} / \mathrm{Pt}$ nanowire array in Figure $7 \mathrm{~d}-\mathrm{f}$ provides a typical example. Followed the steps shown in Figure 7e, the photo-generated electrons and holes are spatially separated by the electrical potential gradient from the metal-semiconductor Schottky junction, and then migrate to the oxidation and reduction electrocatalysts to carry out the desired redox reactions. The solar-to-hydrogen efficiency can reach $0.9 \%$ [133]. (3) Z-scheme design can also contribute to the design of single-unit device. Due to the earth abundance, $\mathrm{Si}$ and $\mathrm{TiO}_{2}$ were chosen as the hydrogen-generating photocathode and oxygen-generating photoanode, respectively. Photo-generated electrons and holes can be effectively separated by the electric field of the $p-n$ junction according to the band diagram in Figure $7 \mathrm{~h}$. The $\mathrm{Si} / \mathrm{TiO}_{2}$ nanotree array shown in Figure $7 \mathrm{~g}$ can produce hydrogen in the rate of $875 \mu \mathrm{mol} \mathrm{h} \mathrm{h}^{-1}$ per gram of materials (Figure 7i), and while energy conversion efficiency can achieve $0.12 \%$ under 1.5 sun illumination [135]. It is more worth mentioning that a novel binary AAO template offers more promise for realizing a side-by-side unit for Z-scheme-based single-unit device, where anode $\left(\mathrm{TiO}_{2}\right)$ and cathode $\left(\mathrm{Cu}_{2} \mathrm{O}\right)$ can harvest solar illumination and participate in separated electrochemical steps for either hydrogen production or oxygen evolution. Each unit consists of one $\mathrm{TiO}_{2}$ nanowire as anode and one $\mathrm{Cu}_{2} \mathrm{O}$ nanowire as cathode. Although no direct contact between the two constituents, the novel connections through conductive substrate offers the possibilities to complete full water splitting under solar illuminations (Figure 71) based on the band diagram in Figure 7k [32]. 


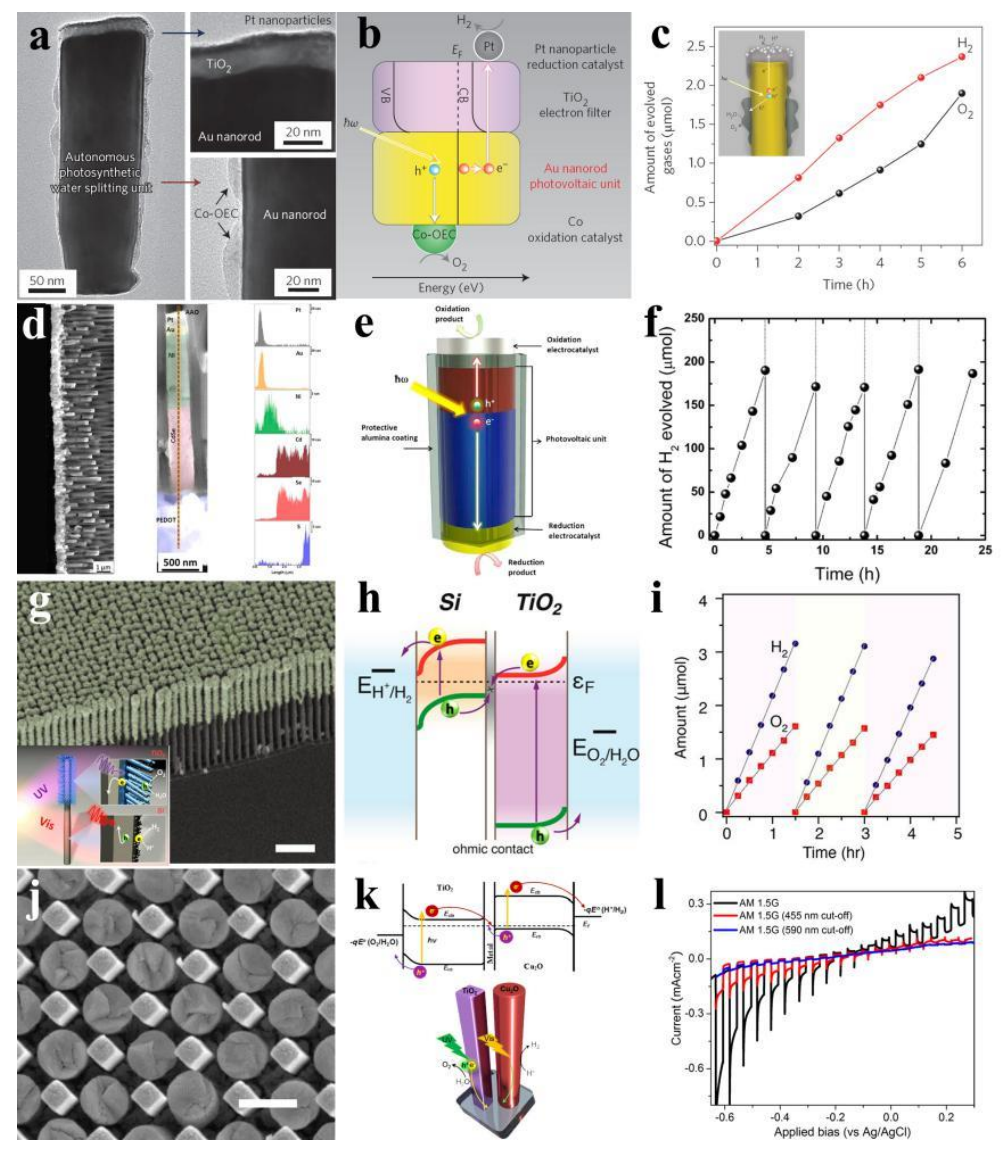

Figure 7. Typical examples of heterogeneous nanostructure arrays with 'Single-unit device' working mode. (a-c) Single-unit device design based on surface plasmon resonance (SPR) effect. (a) The transmission electron micrograph (TEM) of single unit (left) and magnified TEM views of the $\mathrm{Pt} / \mathrm{TiO}_{2}$ cap (top right) and the $\mathrm{Co}-\mathrm{OEC}$ (bottom right), (b) Energy level diagram of an individual unit with the proposed processes occurring in its various parts and in energy space, (c) $\mathrm{O}_{2}$ and $\mathrm{H}_{2}$ evolution under $300 \mathrm{~mW} \mathrm{~cm} \mathrm{~cm}^{-2}$ of white light (AM 1.5). Reproduced with permission. [134] Copyright 2013, Springer Nature. (d-f) Single-unit device design based on photovoltaics. (d) Cross-sectional SEM image and high-magnification SEM image of the individual unit, (e) Schematic of a cross-section of an individual unit, (f) $\mathrm{H}_{2}$ evolution under UV-filtered illumination $(\lambda>410 \mathrm{~nm})$. Reproduced with permission. [133] Copyright 2013, American Chemical Society. (g-i) Single-unit device design based on Z-scheme. (g) Structural characterization of the $\mathrm{Pt} / \mathrm{TiO}_{2}$ nanotree array with structural schematics, scale bars:10 $\mu \mathrm{m}$; (h) Energy band diagram, (i) The evolution of 
$\mathrm{H}_{2}$ and $\mathrm{O}_{2}$ under simulated sunlight of $150 \mathrm{~mW} \mathrm{~cm}^{-2}$ (1.5 suns). Reproduced with permission. [135] Copyright 2013, American Chemical Society. (j) Structural characterization of $\mathrm{TiO}_{2} / \mathrm{Cu}_{2} \mathrm{O}$ binary nanowire array, scale bars: $400 \mathrm{~nm}$, (k) Energy band diagram and structural schematics, (1) Chopped current densities under illumination of AM 1.5. Reproduced with permission. [32] Copyright 2017, Springer Nature.

\section{Application of heterogeneous nanostructure arrays for electrochemical energy storage}

\subsection{Alkali-ion battery}

\subsubsection{Function-Function}

Integrating two electrochemically active constituents in one unit can directly enhance the ability to store charge, i.e., increasing capacity, by taking the contributions of both functional constituents. The design of such kind of electrodes however is more than simply for increasing the capacity. Long-term structural stability and high rate capability also are crucial factors that need to be taken into considerations. This is extremely important for electrodes that undergo conversion and/or alloying mechanisms where reconstruction of crystal structure and large volume change are involved. It has been proven effective that introducing one active material can reduce the pulverization and agglomeration of other active materials, and enhance the electronic and/or ionic conductivity at the same time. A comprehensive improvement of the electrochemical performance thereby can be achieved using the two-material integrated electrodes comparing with the single-material counterparts. Song et al. reported vertically aligned Si-Ge double-layered nanotube (Si/Ge DLNT) arrays as anodes in LIBs (Figure 8a,8b) [136]. Both Si and Ge are electrochemically active towards Li. In the DLNT structure, the Ge out layer has two functions. First, it minimizes the mechanical stress and electrochemical resistance of the inner Si layer 
during lithiation. Second, it possesses higher electronic conductivity, higher lithium ionic diffusivity, and smaller volume change than the inner Si layer. The Si/Ge DLNT electrode exhibited an area capacity of $1.2 \mathrm{mAh} \mathrm{cm}^{-2}$ and delivered a charge capacity of $61 \%$ at the $3 \mathrm{C}$ rate, both of which are 2 -fold higher than those of the Si nanotube (NT) electrode (Figure 8c). The maximum hoop strain in the $\mathrm{Si} / \mathrm{Ge}$ DLNT electrode was calculated to be largely reduced comparing with the Si NT electrode, which was experimentally verified by the fact that the $\mathrm{Si} / \mathrm{Ge}$ DLNT electrode retained its original shape without mechanical degradation after cycling. Wang et al. fabricated $\mathrm{Co}_{3} \mathrm{O}_{4} / \mathrm{CuO}$ nanowire (NW) heterostructure arrays which consists of the well-aligned $\mathrm{CuO}$ nanowire cores, highly porous branch $\mathrm{Co}_{3} \mathrm{O}_{4}$ nanosheet shells, and ordered porous array configuration [137]. The $\mathrm{Co}_{3} \mathrm{O}_{4} / \mathrm{CuO}$ electrode as the LIB anodes showed no obvious capacity fading in the first 140 cycles at $0.2 \mathrm{~A} \mathrm{~g}^{-1}$ and delivered a $90.9 \%$ capacity retention $\left(1191 \mathrm{mAh} \mathrm{g}^{-1}\right)$ after 200 cycles. It also exhibited capacities of 805 and $580 \mathrm{mAh} \mathrm{g}^{-1}$ at 1 and $2.5 \mathrm{~A} \mathrm{~g}^{-1}$, respectively. All the results were much improved comparing with those of the individual $\mathrm{Co}_{3} \mathrm{O}_{4}$ and $\mathrm{CuO} \mathrm{NW}$ arrays. The authors attributed the results to the synergistic contribution from the $\mathrm{CuO} \mathrm{NW}$ core and the $\mathrm{Co}_{3} \mathrm{O}_{4}$ branches, the porous array configuration that increases the amount of contact areas between electrode and electrolyte, and the hierarchically open structure that provides sufficient space to buffer the volume change during cycling. The synergistic effect was also observed in the $\mathrm{Fe}_{3} \mathrm{O}_{4}$ quantum dots (QDs) decorated $\mathrm{MoS}_{2}$ nanosheet arrays as SIB anodes (Figure 8d) [138]. The interconnected $\mathrm{MoS}_{2}$ nanosheets provide flexible substrates for the decoration of the QDs and accommodate the volume change of the QDs during cycling. The $\mathrm{Fe}_{3} \mathrm{O}_{4} \mathrm{QDs}$ as return act as spacers to stabilize the composite structure by ensuring the active surfaces of the nanosheets to be accessible for electrolyte penetration. In addition, the ultra-small size of the QDs shortens the diffusion path of sodium ions and electrons. As a result, the composite delivered capacities of 468 and $231 \mathrm{mAh} \mathrm{g}^{-1}$ at 0.1 and $3.2 \mathrm{~A} \mathrm{~g} \mathrm{~g}^{-1}$, respectively. It also retained $72.5 \%$ of the original capacity after 300 cycles at $0.1 \mathrm{~A}$ $\mathrm{g}^{-1}$ (Figure 8e,8f). The composite therefore exhibited a significant improvement of the 
overall electrochemical performance over the $\mathrm{MoS}_{2}$ nanosheet arrays.

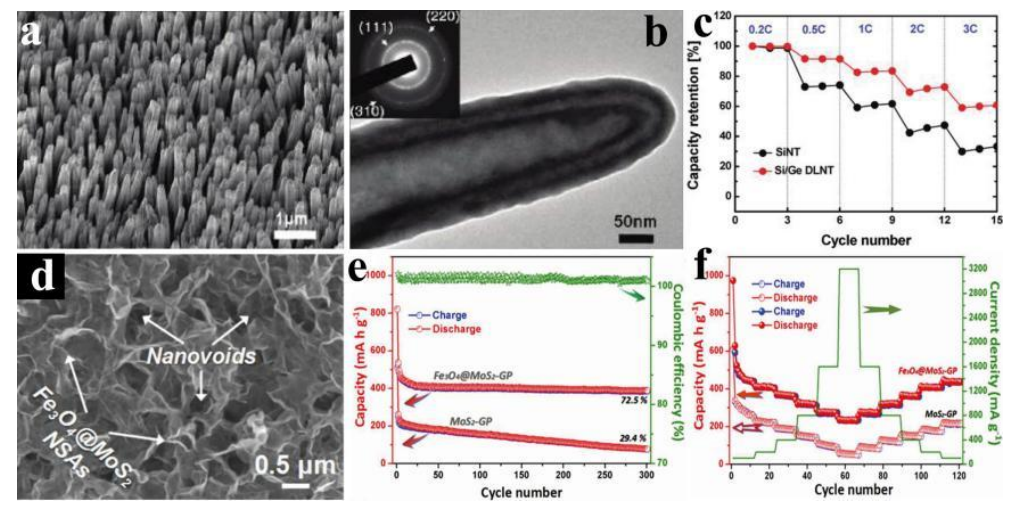

Figure 8. Typical examples of heterogeneous nanostructure arrays with 'Function-Function' working mode for alkali-ion battery. (a-c) SEM (a) and TEM (b) images of the vertically aligned Si-Ge double-layered nanotube (DLNT) arrays on a stainless steel substrate. Inset of (b) shows selective area electron diffraction (SAED) pattern. (c) Rate capabilities of Si nanotube (NT) and Si/Ge DLNT array electrodes. Reproduced with permission. [136] Copyright 2012, American Chemical Society. (d) SEM image of $\mathrm{Fe}_{3} \mathrm{O}_{4}$ quantum dots (QDs)@ $\mathrm{MoS}_{2}$ nanosheets grown on a graphite paper (GP). Cycling performance (e) and rate capability (f) of the $\mathrm{Fe}_{3} \mathrm{O}_{4}$ QDs@MoS 2 -GP and $\mathrm{MoS}_{2}$-GP composite electrodes. Reproduced with permission. [138] Copyright 2017, Royal Society of Chemistry.

\subsubsection{Function-Assistance}

A heterogeneous electrode also can consist of an electrochemically active material as a functional constituent and an electrochemically inactive material (or a miner contributor to the battery capacity in some cases) as an assistance constituent. The rationale behind such kind of electrode design always centers at improving ion and electron transport as well as enhancing structural stability of the electrodes. A secondary constituent could be integrated into the electrodes as either an inner scaffold or an external coating layer. The form of inner scaffold generally refers to using the arrays of conductive materials as current collector of the electrodes. For the 
former, a wide range of conductive materials has been employed as current collectors so far. These materials include metals ( $\mathrm{Ni}, \mathrm{Al}, \mathrm{Cu}, \mathrm{Fe}, \mathrm{Co}, \mathrm{Ag}, \mathrm{Au}, \mathrm{Ti}$, and $\mathrm{Si}$ ) carbons, (CNTs, graphene, soft carbon, and hard carbon), and compounds.( $\mathrm{TiO}_{2}, \mathrm{TiSi}_{2}$, and ITO) Due to the diversity of the materials, we will discuss this type of electrodes, i.e., inner conductive scaffold and surface coated active materials, from the perspective of the dimension of the scaffold, where the design rationale could be demonstrated in the scaffold with different dimensions. Xu et al. proposed highly ordered 1D conductive scaffold with high regularity and strictly vertical alignment of the unit structure [60]. These features enable high ion accessibility in the interspace between the neighboring units and fast electron transport along the vertical direction in the arrays (Figure 9a). The authors fabricated highly ordered $\mathrm{Ni}^{-\mathrm{TiO}_{2}}$ core-shell nanowire arrays (NTNAs) as an example based on the highly ordered Ni nanopillar arrays as the conductive inner scaffold (Figure 9b). Used as SIB anodes, the HNAs delivered a capacity of $\sim 200 \mathrm{mAh} \mathrm{g}^{-1}$ after 100 cycles at $50 \mathrm{~mA} \mathrm{~g}^{-1}$ and retained $\sim 95 \mathrm{mAh} \mathrm{g}^{-1}$ at $5 \mathrm{~A} \mathrm{~g}^{-1}$ (Figure 9c). Comparing with 1D conductive scaffold, 2D conductive scaffold can provide multiple electron transport pathways and thereby afford better structural stability of the electrode (Figure 9d). Xie et al. conducted a systematic study to compare the LIB performance of the nanoarray electrodes using $1 \mathrm{D}$ and $2 \mathrm{D}$ conductive scaffolds [139]. Both electrodes shared the same composition where $\mathrm{TiSi}_{2}$ nanowires or nanonets (Figure 9e) as the current collector and Si as the active material. The nanonet-based nanoarrays exhibited higher capacity and retention than the nanowire-based ones at different operation conditions, i.e., $90 \%$ vs. $80 \%$ retention after 100 cycles at $6 \mathrm{~A} \mathrm{~g}^{-1}$ (Figure 9f), which was ascribed to the 2D connectivity. A 3D conductive scaffold can offer both high electronic conductivity and fast ion transport pathways within the whole scaffold. Zhang et al. constructed a self-assembled 3D bicontinuous nanoarchitecture consisting of a 3D Ni scaffold and $\mathrm{MnO}_{2}$ coated on the surface of the scaffold (Figure 9h) [140]. The nanoarchitechture delivered very large LIB battery charge and discharge rate with minimal capacity loss. $76 \%$ and $38 \%$ of the original cathode capacity were retained at the rates as high as 
185 and $1114 \mathrm{C}$, respectively (Figure 9i). Furthermore, a full cell coupled with a graphite anode was able to be $90 \%$ charged in 2 minutes, demonstrating that the bicontinuous 3D scaffold can enable the optimization to match the characteristic kinetics of a given battery chemistry. A great challenge of using inner conductive scaffold is that the improvement of ion and electron transport should be achieved in a thick electrode, meaning with a high mass loading of active materials. Inspired by the multi-channeled natural wood, Chen et al. developed an ultra-thick 3D carbon framework as a current collector with high conductivity, low tortuosity, and low deformability [141]. Electrons can move fast along the aligned channels of the carbonized matrix and ions can diffuse fast in the electrolyte-filled gaps at the edge of the channels, thereby contributing to a high electronic conductivity and high ionic diffusivity. A 3D electrode consisting the carbon framework and $\mathrm{LiFePO}_{4}$ was fabricated as an example. The electrode has a thickness of $80 \mu \mathrm{m}$ and a mass loading of $60 \mathrm{mg} \mathrm{cm}$, for which easy deformability in the form of electrode cracking and detachment from the Al current collector was observed in a conventional electrode prepared by slurry coating method. The ultra-thick 3D electrode delivered a high capacity of $7.6 \mathrm{mAh} \mathrm{cm} \mathrm{c}^{-2}$ (95 $\mathrm{Ah} \mathrm{L}^{-1}$ based on volume) at $0.5 \mathrm{~mA} \mathrm{~cm}$-2 and energy density of $26 \mathrm{mWh} \mathrm{cm}^{-2}$ (323 $\mathrm{Wh} \mathrm{L}^{-1}$ based on volume). 

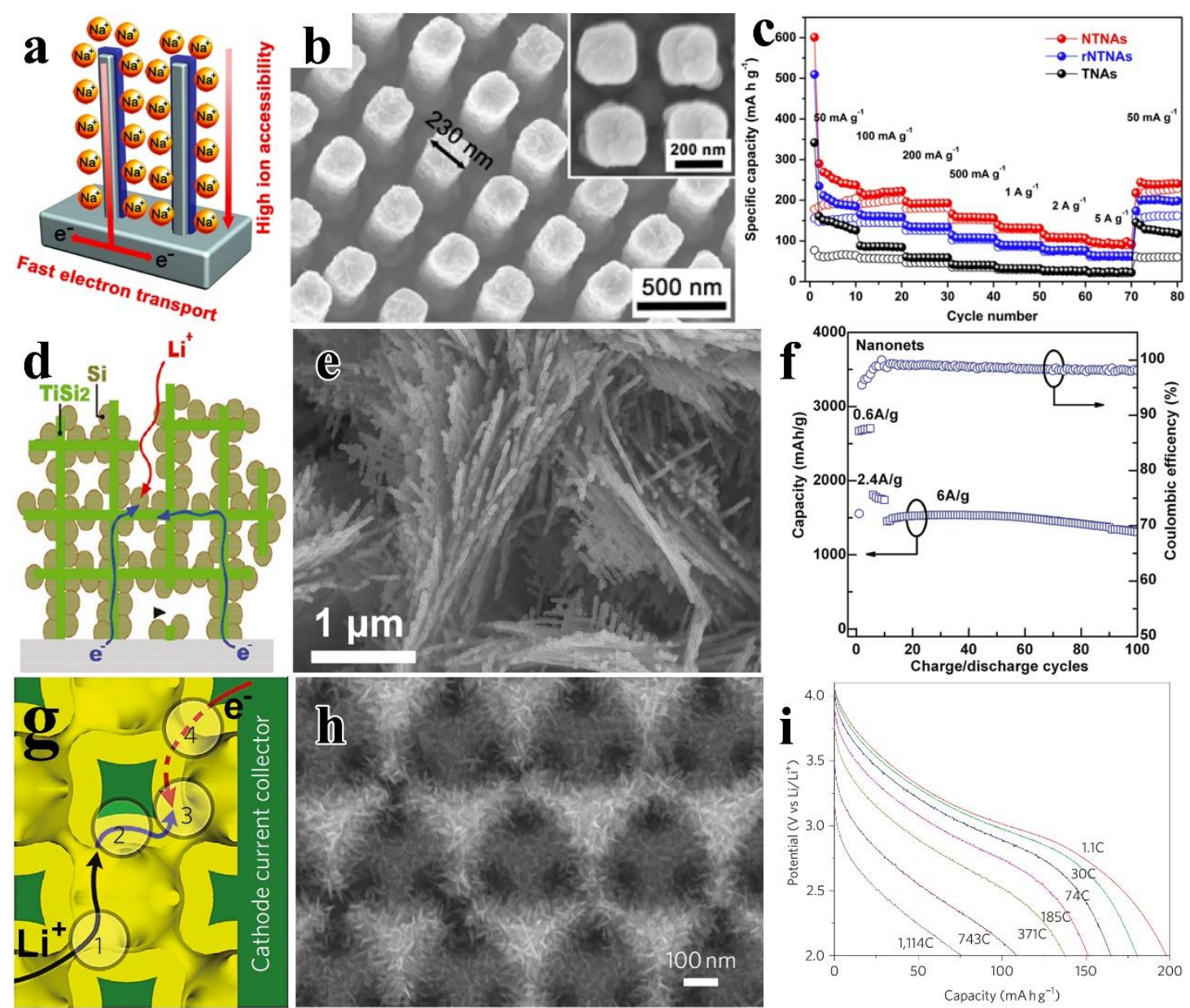

Figure 9. Typical examples of heterogeneous nanostructure arrays with 'FunctionAssistance' working mode for alkali-ion battery by means of multi-dimensional current collectors. (a-c) Illustration and SEM image of the highly ordered nanoarrays using a 1D conductive scaffold, (c) Rate capability of the highly ordered highly ordered $\mathrm{Ni}^{-\mathrm{TiO}_{2}}$ core-shell nanowire arrays (NTNAs), randomly oriented NTNAs (rNTNAs), and $\mathrm{TiO}_{2}$ nanotube arrays (TNAs). Reproduced with permission. [60] Copyright 2015, American Chemical Society. (d-f) Illustration and scanning electron microscope (SEM) image of the $\mathrm{TiSi}_{2} / \mathrm{Si}$ HNAs using a $2 \mathrm{D}$ conductive scaffold, and cycling performance (f) at various current densities of the $\mathrm{TiSi}_{2} / \mathrm{Si}$ HNAs. Reproduced with permission. [139] Copyright 2011, American Chemical Society. (g-i) Illustration and SEM image of the bicontinuous $\mathrm{Ni}-\mathrm{MnO}_{2}$ heterostructure architecture using a 3D conductive scaffold, (i) discharge profiles at various $\mathrm{C}$ rates of the Ni-lithiated $\mathrm{MnO}_{2}$ composite electrode. Reproduced with permission. [140] Copyright 2011, Springer. 
Coating the electrochemically active material with an external layer is another way to form heterostructure array electrodes. Most of the coating materials are carbons (CNTs, graphene, graphitic carbon, hard carbon, and doped carbon) and conductive polymer, where their conductive nature can improve ion and electron transport and enhance the structural stability of the electrodes. The latter is very challenging for the active materials that use conversion and/or alloying mechanisms and experience large volume change during cycling, as we discussed in the previous section. Therefore, the conductive coating is commonly seen in the active materials of metals and oxides. Xie et al. fabricated core-sheath structured Sn@CNT nanopillar arrays vertically on carbon paper [142]. The CNT sheath can enhance the electrical conductivity of the electrode and sustain the integrity of the electrode by provide buffering capability for the volume change during Na-Sn alloying. The nanopillar arrays were used as SIB anodes and delivered high areal capacities of 550, 377 and $299 \mu \mathrm{Ah} \mathrm{cm}^{-2}$ at 50, 500 and $1000 \mu \mathrm{A} \mathrm{cm}^{-2}$, respectively. Carbon coating layer can be doped with nitrogen so that the $\mathrm{N}$-doped carbon layer not only enhances the electrical conductivity and sustains the integrity of the electrode but also contributes to the capacity owing to the abundant defects and active sites created by the N-dopant. Tan et al. fabricated cable-like $\mathrm{CuO} / \mathrm{CN}_{\mathrm{x}}$ core-shell nanowire arrays on the foil (Figure 10a), net and grid substrates and used them as LIB anodes [143]. The $\mathrm{CuO} / \mathrm{CN}_{\mathrm{x}}$ arrays on the grid substrate with a $\mathrm{N}$-doping level of $\sim 20$ at\% exhibited a capacity of $669.0 \mathrm{mAh} \mathrm{g}^{-1}$ at $50 \mathrm{~mA} \mathrm{~g}^{-1}$ and a retention of $83.3 \%$ after 200 cycles (Figure $10 \mathrm{~b}$ ). Both values were almost as twice much as those of the bare $\mathrm{CuO}$ arrays. The mechanical buffering capability of the $\mathrm{CN}_{\mathrm{x}}$ layer was confirmed by the in-situ TEM measurement of the charge-discharge cycle on a single core-shell nanocable. The $\mathrm{CuO}$ core was observed to experience a volume expansion by $\sim 93 \%$, however the volume of the entire nanocable showed no evident expansion (Figure 10c), indicating a good structural integrity owing to the mechanically stable $\mathrm{CN}_{\mathrm{x}}$ shell. Choices of conductive polymers as coating layers are less than carbons. The reported polymer coating layers so far include poly(3,4-ethylenedioxythiophene) [PEDOT], poly(ethylene oxide) [PEO], 
polypyrrole (PPy), polyaniline (PANI), and poly(methyl methacrylate) [PMMA]. Cui's group reported PEDOT coating improved the cycling stability of Si NW electrodes in LIBs owing to its high electrical conductivity (n-doped state, $0.1 \mathrm{~S} \mathrm{~cm}^{-1}$ ) and good electrochemical stability [144]. A capacity of $2500 \mathrm{mAh} \mathrm{g}^{-1}$ was retained after 100 cycles for the PEDOT-coated Si NWs. This was $80 \%$ of the initial capacity comparing with $30 \%$ retention in the case of bare Si NWs under the same cycling conditions. Chao et al. coated a mesoporous thin layer of PEDOT on the $\mathrm{V}_{2} \mathrm{O}_{5}$ nanobelt arrays (Figure 10d,10e) directly grown on the 3D ultrathin graphite foam (UGF) [145]. The thin layer can not only facilitate the electron transfer around $\mathrm{V}_{2} \mathrm{O}_{5}$ but also decrease the polarization and improve the reaction kinetics. As a result, ultrafast and stable Li storage was achieved by delivering capacities of 265 and 168 $\mathrm{mAh} \mathrm{g}^{-1}$ at the 5 and $60 \mathrm{C}$ rates (Figure 10f), respectively, and maintaining $98 \%$ of the capacity up to 1000 cycles. Although most of the coating materials are carbons and conductive polymers, there have been reports of using compounds as the coating layers to address specific issues beyond charge transport. For instance, intercalation-type cathode materials tend to dissolve in electrolytes, which often leads to poor cycle performance. This issue is even more detrimental at an elevated temperature. Guo et al. proposed to coat a layer of $\mathrm{Li}_{2} \mathrm{CO}_{3}$ to solve the dissolution issue (Figure 10g) [146]. $\mathrm{Li}_{2} \mathrm{CO}_{3}$ is a constituent of the solid electrolyte interface (SEI) layer and thus difficult to be electrochemically reduced at both room temperature and a high temperature, for the latter $\mathrm{O}_{2}$ evolution could be minimized or avoided. The $\mathrm{Li}_{2} \mathrm{CO}_{3}$ coated $\mathrm{LiMnO}_{2}$ (LMO) cathode not only exhibited significantly higher capacities than the bare LMO counterpart at the same current densities at room temperature, it also showed well-balanced performance at $60^{\circ} \mathrm{C}$, delivering capacities of 207 and $128 \mathrm{mAh} \mathrm{g}^{-1}$ at the rates of 0.5 and $5 \mathrm{C}$ (Figure 10h,10i), respectively, and retaining $79 \%$ capacity after 400 cycles. As discussed in the previous section, the conductive inner scaffold provides fast electron transport and enhances the structural stability of the electrode. It naturally leads researchers to the considerations that the coexistence of the conductive inner scaffold and external coating layer could offer the 
combined benefits that each form can offer. In this regard, it has been several examples of arrayed electrodes in a sandwich structure where a carbon material and an active material were layer-by-layer arranged in a radial or vertical direction. C@Si@C nanoarrays [147], C/Sn/Ni/TMV nanoforest [148], and multilayer nanoassembly of Sn nanopillar arrays and graphene layers [4] have been reported in LIBs so far to demonstrate that the sandwich structure may serve as a guide for many emerging electrode systems.

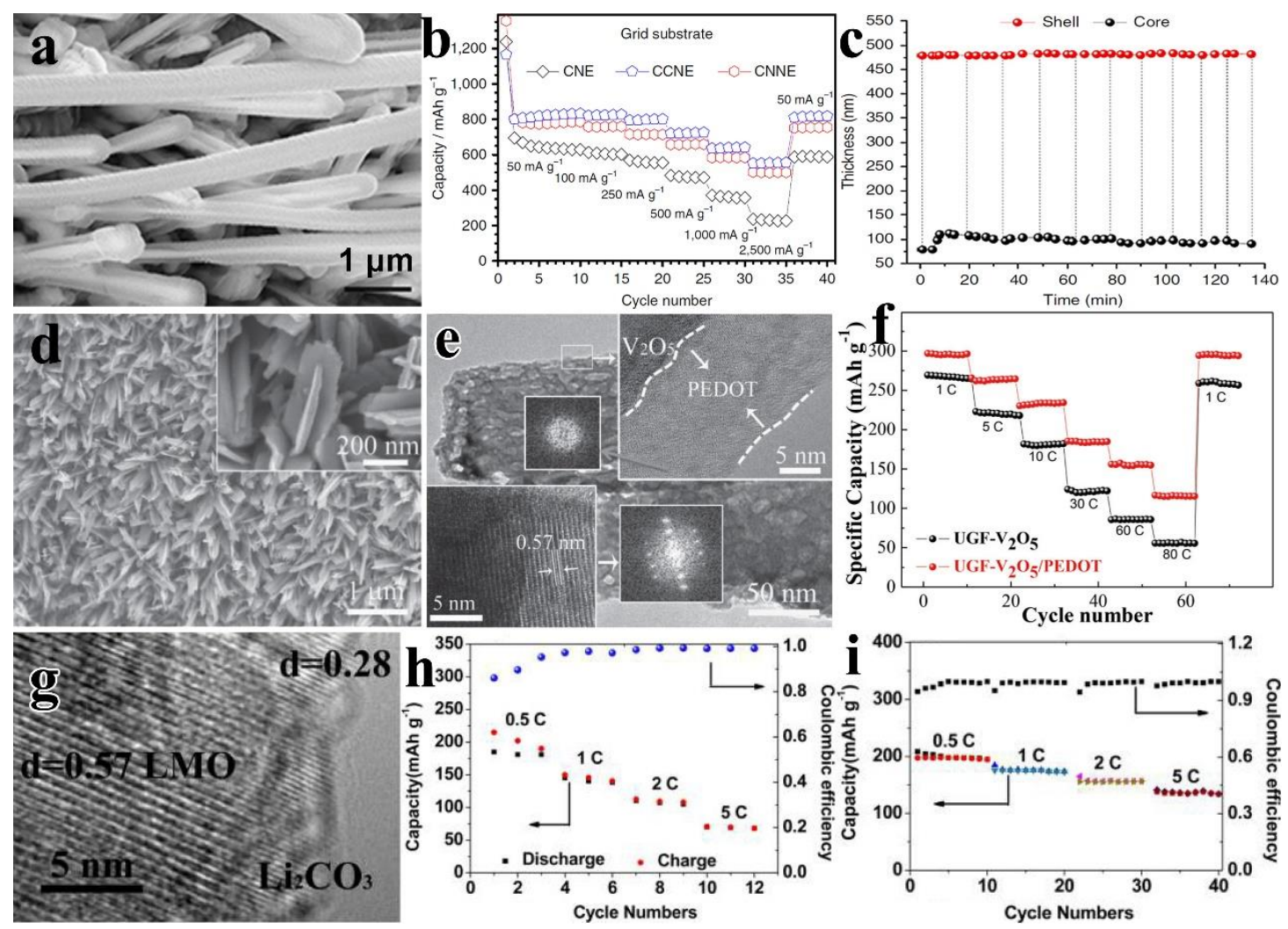

Figure 10. Typical examples of heterogeneous nanostructure arrays with 'FunctionAssistance' working mode for alkali-ion battery by means of surface coating. (a) Scanning electron microscope (SEM) image of the $\mathrm{CuO} / \mathrm{CN}_{\mathrm{x}}$ nanocable electrode (CCNE). (b) Rate capability of the CCNE, CuO nanowire electrode (CNE), and $\mathrm{Cu} / \mathrm{CN}_{\mathrm{x}}$ nanocable electrode (CNNE). (c) Thickness variation curves of the CCNE nanocable during lithiation-delithiation cycles. Reproduced with permission. [143] Copyright 2016, Springer. SEM (d) and transmission electron microscope (TEM) (e) images of the ultrathin graphite foam (UGF)- $\mathrm{V}_{2} \mathrm{O}_{5} / \mathrm{PEDOT}$ core/shell nanobelts. Inset 
of (e) are HRTEM images and FFT patterns. (f) Rate capability of the UGF- $\mathrm{V}_{2} \mathrm{O}_{5} / \mathrm{PEDOT}$ and UGF- $\mathrm{V}_{2} \mathrm{O}_{5}$ nanobelts. Reproduced with permission. [145] Copyright 2014, Wiley-VCH. (g) HRTEM image of the LMO@ $\mathrm{Li}_{2} \mathrm{CO}_{3}$ nanosheet. Rate capability of the LMO electrode at $20^{\circ} \mathrm{C}(\mathrm{h})$ and $\mathrm{LMO} @ \mathrm{Li}_{2} \mathrm{CO}_{3}$ electrode at $60^{\circ} \mathrm{C}$. Reproduced with permission. [146] Copyright 2016, American Chemical Society.

\subsubsection{Single-unit device}

HNAs possess great advantages as battery electrodes as we have discussed, regardless the forms of how constituents integrate with each other. One of the major advantages is the improved charge transport that plays a critical role in determining the battery performance. One can imagine that the battery performance could be ultimately maximized if a battery consists of multiple single units where each unit embeds all constituents, i.e., cathode, anode, and electrolyte, because of close proximity of electrodes between ultrathin electrolyte layers and ultrashort ion diffusion distances between the electrodes. This kind of all-in-one batteries could provide high energy and power density in a small footprint area and result in the ultimate miniaturization of energy devices. Constructing all-in-one batteries is technically challenging because of the integration of two electrodes in one unit and the confinement of the electrolyte. Thus, there have been limited examples of LIBs but none of SIBs. Liu et al. fabricated an all-in-one LIB by utilizing the vertically nanotubular pores in an AAO template [149]. The $\mathrm{Ru}$ current collector and electroactive $\mathrm{V}_{2} \mathrm{O}_{5}$ were conformably deposited at the two ends of the nanopores, forming a head-to-head configuration of single unit, for which controlling the deposition depth of the materials allows the electrical isolation between the two ends (Figure $11 \mathrm{a}, 11 \mathrm{~b}$ ). With pristine $\mathrm{V}_{2} \mathrm{O}_{5}$ as cathode, lithiated $\mathrm{V}_{2} \mathrm{O}_{5}$ as anode, and liquid electrolyte confined inside of the pores, the battery delivered capacities of 178 and $82 \mathrm{mAh} \mathrm{g}^{-1}$ at the rates of 1 and $150 \mathrm{C}$ (Figure 11c), respectively, and retained more than $80 \%$ of the initial capacity after 1000 cycles. The battery performance fully demonstrated the design of integrated nanotubular 
electrodes that guarantees fast charge transport. Ajayan's group employed the similar head-to-head configuration to construct a single nanowire battery with the anode (Ni-Sn) and cathode (polyaniline) nanowires packaged within liquid electrolyte-soaked polymer (PEO) core-shell separator [150]. The parallel array of the nanowire batteries however showed a relatively low areal capacity and unsatisfactory cyclability, presumable due to the very thin layer the soaked PEO that could result in the depletion of Li ions during battery operation.
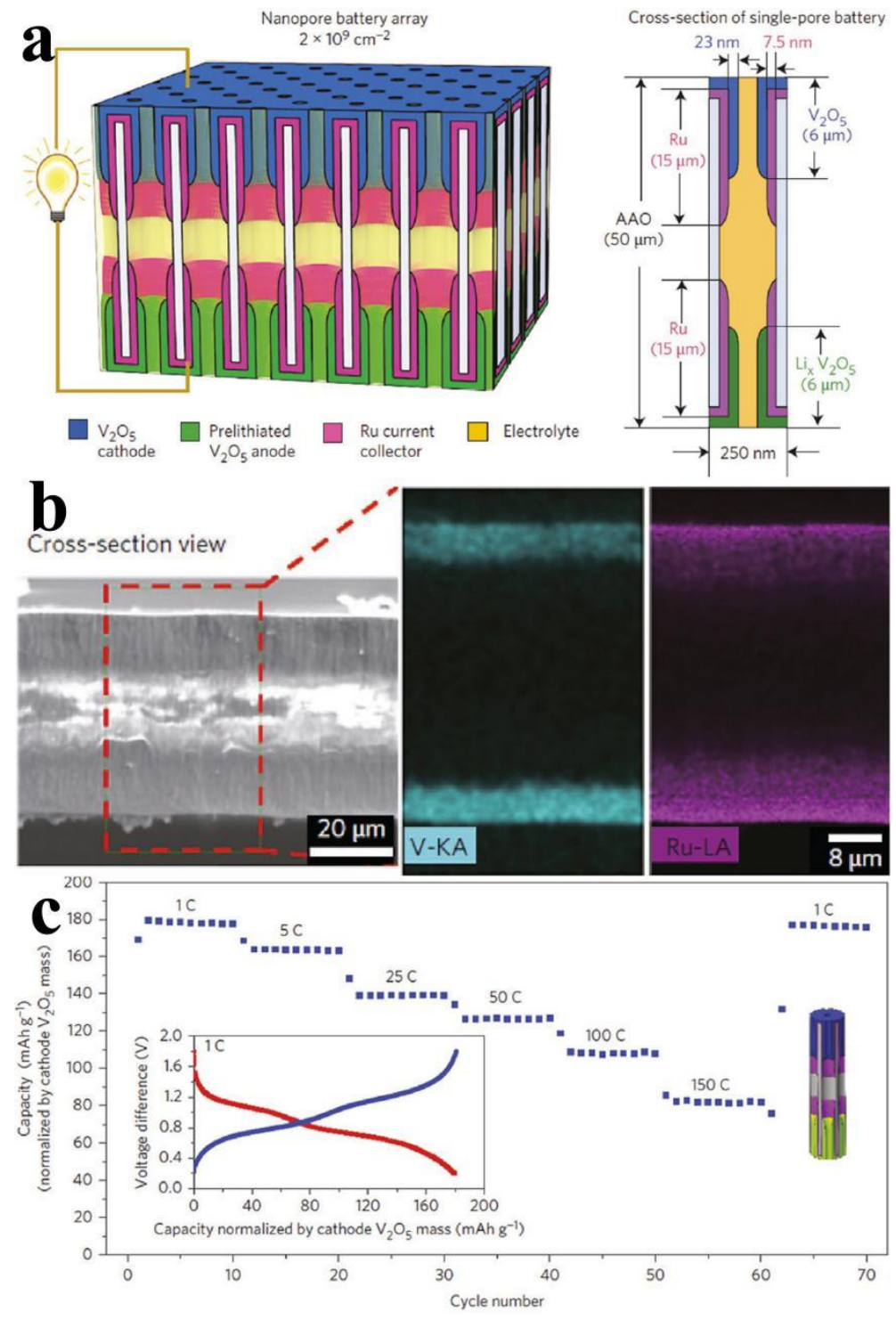

Figure 11. Typical examples of heterogeneous nanostructure arrays with 'Single-unit device' working mode for alkali-ion battery. (a) Illustration of the parallel nanopore 
battery arrays and cross-section of a single-pore battery. (b) Energy-dispersive X-ray spectroscopy (EDS) mapping of device cross-section, demonstrating its symmetrical manner. (c) Rate capability of the nanopore battery arrays. Inset is the charge-discharge profiles at 1 C. Reproduced with permission. [149] Copyright 2014, Springer Nature.

\subsection{Supercapacitor}

The major challenge of supercapacitors, when comparing with batteries, is the insufficient energy density. The energy density of a supercapacitor is proportional to the capacitance $(C)$ and square of the cell potential $(V)$, according to the equation of $E$ $=0.5 C V^{2}$. Therefore, increasing the capacitance of the electrode and/or the cell potential can efficiently enhance the energy density. For the former, combining two electroactive materials with high pseudocapacitance is a widely employed strategy; while for the latter, adopting asymmetric electrode configuration and developing electrolytes with wide electrochemically stable window are generally considered as practical pathways. There have been many great reviews summarizing the asymmetric electrode configuration and the development of electrolytes [151-154]. In line with the focus of this review, we will discuss the heterostructure of the single electrode.

\subsubsection{Function-Function}

The design of combining two electroactive materials in one electrode primarily aims at combining the high pseudocapacitance of both materials, but the composites also offer synergetic effect or multi-functionalities. It is critical that one of the materials possesses large surface area in order to composite with a large amount of the other material, for which the synergetic effect takes place. Xia et al. synthesized transition metal oxide core/shell nanoarrays on a variety of substrates including transparent conducting glass, nickel foil, and nickel foam [155]. Taking $\mathrm{Co}_{3} \mathrm{O}_{4} / \mathrm{NiO}$ core/shell nanoarrays as an example (Figure 12a), the heterogeneous electrode 
maintained $85 \%$ of the capacitance (from 452 to $384 \mathrm{~F} \mathrm{~g}^{-1}$ ) when the rate changed from 2 to $40 \mathrm{~A} \mathrm{~g}^{-1}$, being much higher than those for the $\mathrm{Co}_{3} \mathrm{O}_{4}$ naowire arrays $(76.7 \%)$ and $\mathrm{NiO}$ nanoflake arrays (66.6\%) (Figure 12b). The heterogeneous electrode also exhibited great cyclability by delivering a capacitance of $853 \mathrm{~F} \mathrm{~g} \mathrm{~g}^{-1}$ after 6000 cycles at $2 \mathrm{~A} \mathrm{~g}^{-1}$, comparing with $642 \mathrm{~F} \mathrm{~g}^{-1}$ for the $\mathrm{Co}_{3} \mathrm{O}_{4}$ arrays and $178 \mathrm{~F} \mathrm{~g}^{-1}$ for the $\mathrm{NiO}$ arrays (Figure 12c). The authors attributed the enhance performance to the synergetic effect of the heterostructure. First, the porous $\mathrm{Co}_{3} \mathrm{O}_{4}$ nanowires and the well-separated $\mathrm{NiO}$ nanoflakes make both core and shell fully accessible to the electrolyte, enhancing ion and electron diffusion. Second, the arrayed configuration ensures good adhesion to the substrate, providing mechanical stability over long-term cycling where the core/shell structure remained intact after 6000 cycles. These synergetic effects have been reported by a great number of literatures of the HNAs consisting of Ni-, Co-, and Mn-based oxides and/or binary oxides. Zhu et al. reported another kind of synergetic effect to enhance capacitance by optimizing the phase combination in the array structure [156]. Bi-phase self-branched $\mathrm{MnO}_{2}$ (SBM) nanoarray structure was fabricated where the $\alpha-\mathrm{MnO}_{2}$ nanowire cores and the $\delta-\mathrm{MnO}_{2}$ nanoflake branches have different crystal structures and capacitive properties. The SBM composite electrode exhibited higher capacitance than both $\mathrm{MnO}_{2}$ nanowires and nanoflakes at different scan speeds. Besides the increased surface area, the authors ascribed the capacitance enhancement to the lattice defects caused by the poor-defined crystallographic interfaces between the two phases, where the defects can lead to further exposure of electrochemical active sites. Multi-functionalities can be realized if one of the electroactive materials has high surface area and is conductive at the same time, providing capacitance, high loading of another electroactive material, and facile charge transport simultaneously. Highly conductive $\mathrm{NiCo}_{2} \mathrm{~S}_{4}$ nanoarrays have been demonstrated to serve not only as a good pseudocapacitive material but also as a $3 \mathrm{D}$ conductive scaffold capable of fast electron conduction and ion diffusion [157, 158]. Xiao et al. prepared $\mathrm{NiCo}_{2} \mathrm{~S}_{4}$ nanotube arrays grown on a flexible carbon fiber paper and deposited a series of electroactive materials, including $\mathrm{Co}_{\mathrm{x}} \mathrm{Ni}_{1-\mathrm{x}}(\mathrm{OH})_{2}$, 
$\mathrm{MnO}_{2}$, and $\mathrm{FOOH}$, on the $\mathrm{NiCo}_{2} \mathrm{~S}_{4}$ scaffold [157]. The $\mathrm{Co}_{\mathrm{x}} \mathrm{Ni}_{1-\mathrm{x}}(\mathrm{OH})_{2} / \mathrm{NiCo}_{2} \mathrm{~S}_{4}$ nanotube array electrode (Figure 12d) showed a high areal capacitance of $2.86 \mathrm{~F} \mathrm{~cm}^{-2}$ at $4 \mathrm{~mA} \mathrm{~cm}^{-2}$, good rate capability of $2.41 \mathrm{~F} \mathrm{~cm}^{-2}$ at $20 \mathrm{~mA} \mathrm{~cm}^{-2}$, and excellent cycling stability of maintaining $96 \%$ of the initial capacitance after 2000 cycles at $10 \mathrm{~mA} \mathrm{~cm}^{-2}$ (Figure 12e,12f). The conductive electroactive materials can also be coated on the surface of another electroactive materials and acts as a protective layer to offer one more function of maintaining the electrode integrity. PPy and PANI are two popular electroactive materials that are applied as a coating layer. Zhou et al. developed an electrode consisting of well-aligned $\mathrm{CoO}$ nanowire array grown on 3D nickel foam with PPy firmly anchored to each nanowire surface to boost the pseudocapacitive performance (Figure 12g) [159]. The electrode architecture takes advantages of the high electroactivity from both the inner $\mathrm{CoO}$ wires and PPy outlayer, the high electronic conductivity of PPy, and the short ion diffusion pathway in the ordered mesoporous $\mathrm{CoO}$ nanowires. These advantages account for a high areal capacitance of $4.43 \mathrm{~F} \mathrm{~cm}^{-2}$ at $1 \mathrm{~mA} \mathrm{~cm}^{-2}$, great rate capability of $1.28 \mathrm{~F} \mathrm{~cm}^{-2}$ at $50 \mathrm{~mA} \mathrm{~cm}^{-2}$, and great cycling stability of delivering $99.8 \%$ capacitance retention after 2000 cycles at $20 \mathrm{~mA}$ $\mathrm{cm}^{-2}$ (Figure 12h,12i). It is worth mentioning that poly(3,4-ethylenedioxythiophene) (PEDOT) was also reported to act as a protective coating layer [160], although it is not an electroactive material.
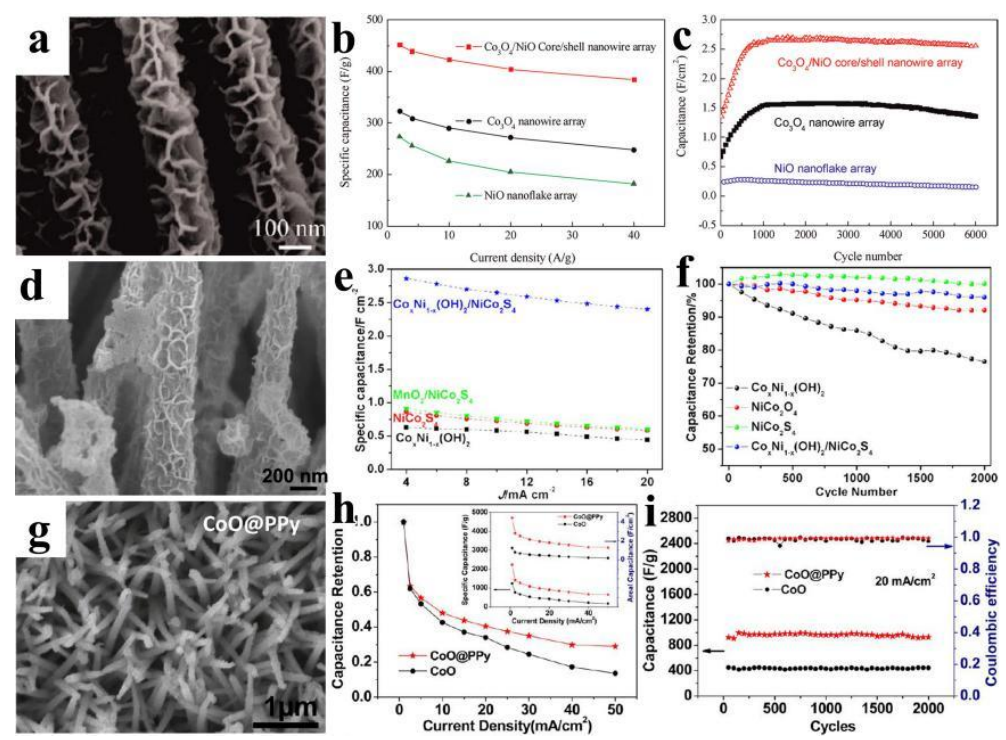
Figure 12. Typical examples of heterogeneous nanostructure arrays with 'Function-Function' working mode for supercapacitor. (a) scanning electron microscope (SEM) image of the $\mathrm{Co}_{3} \mathrm{O}_{4} / \mathrm{NiO}$ core/shell nanoarrays. Specific capacitances at various current densities (b) and cycling performance at $2 \mathrm{~A} \mathrm{~g}^{-1}$ (c) of different array electrodes. Reproduced with permission. [155] Copyright 2012, American Chemical Society. (d) SEM image of the $\mathrm{Co}_{x} \mathrm{Ni}_{1-\mathrm{x}}(\mathrm{OH})_{2} / \mathrm{NiCo}_{2} \mathrm{~S}_{4}$ nanotube arrays. Discharge areal capacitances (e) and cycling performance (f) of different array electrodes. Reproduced with permission. [157] Copyright 2014, American Chemical Society. (g) SEM image of the CoO@PPy nanowire arrays. Capacitance retention as a function of current density (h) and cycling performance (i) of different array electrodes. Inset of (h) is the current density dependence of the areal capacitance and specific capacitance. Reproduced with permission. [159] Copyright 2013, American Chemical Society.

\subsubsection{Function-Assistance}

Many pseudocapacitive materials such as transition metal oxides and hydroxides exhibit high capacitance via fast surface redox reactions, however they generally suffer low electronic conductivity that could result in low energy storage at high power delivery. This issue can be addressed by introducing a conductive constituent in the heterostructure. Such kind of heterostructures often are constructed in the core/shell configuration where the conductive constituent acts as the inner scaffold to provide high electronic conductivity, which shares the same design principle in the case of heterogeneous battery electrodes. Besides the high electronic conductivity, the inner scaffold needs to fulfill another two prerequisites: high surface area to achieve high loading of the electroactive material and high mechanical and chemical stability to reach high cyclability of the electrode. The materials that can be utilized as the inner scaffold spans over a wide range from metals (Ni, $\mathrm{Pt}, \mathrm{Al}, \mathrm{Au}, \mathrm{Mn}, \mathrm{Ti}$, and alloy) to carbon materials (rGO, CNT, and graphene) and further to compounds (TiN, ITO, 
$\mathrm{C}_{3} \mathrm{~N}_{4}, \mathrm{SnO}_{2}, \mathrm{~V}_{2} \mathrm{O}_{3}, \mathrm{WO}_{3}, \mathrm{TiO}_{2}$, and $\mathrm{ZnO}$ ). Again due to the diversity of the materials, we will summarize the core/shell scaffold/electroactive material electrodes from the perspective of the dimension of the inner scaffold. 1D scaffolds are commonly seen as nanowire/nanotube/nanocone/nanospike arrays, and a thin layer of electroactive material is coated on the surface on the 1D scaffold. Xu et al. prepared a vertically aligned Ni nanowire array (NNA) with the diameter ranging from 120 to $170 \mathrm{~nm}$ and length up to $1 \mathrm{~mm}$ (aspect ratio over 8000) as the inner scaffold, followed by electrodepositing $\mathrm{MnO}_{2}$ as cathode (Figure 13a) and PPy as anode for an aqueous asymmetric micro-supercapacitor (NNA-ASC) [161]. The device manifested a high areal capacitance of $135.15 \mathrm{mF} \mathrm{cm}^{-2}$ at $3 \mathrm{~A} \mathrm{~g} \mathrm{~g}^{-1}$ and maintained $92.7 \%$ of the capacitance at $24 \mathrm{~A} \mathrm{~g}^{-1}$ (Figure 13b). The maximum gravimetric energy and power densities were $48.88 \mathrm{Wh} \mathrm{kg}^{-1}$ and $1275 \mathrm{~W} \mathrm{~kg}^{-1}$, respectively. Besides, no capacitance loss was observed after 20000 cycles at $30 \mathrm{~A} \mathrm{~g}^{-1}$ (Figure 13c). The same cathode/anode combination was applied on $\mathrm{SnO}_{2}$ nanotube arrays as inner scaffold by Grote et al. and the prepared device exhibited the energy and power densities of 27.2 $\mathrm{Wh} \mathrm{kg}^{-1}$ and $24.8 \mathrm{~kW} \mathrm{~kg}^{-1}$, respectively [162]. Shang et al. reported a coaxially heterogeneous electrode by using TiN nanotube arrays as the self-standing inner scaffold and $\mathrm{Ni}_{\mathrm{x}} \mathrm{Co}_{2 \mathrm{x}}(\mathrm{OH})_{6 \mathrm{x}}$ as the electroactive material that well-distributed into the TiN nanotubes [137]. The electrode exhibited a high specific capacitance of $2543 \mathrm{~F}$ $\mathrm{g}^{-1}$ at $5 \mathrm{mV} \mathrm{s}^{-1}$, rate capability of $660 \mathrm{~F} \mathrm{~g}^{-1}$ at $500 \mathrm{mV} \mathrm{s}^{-1}$, and great cycling stability with only $6.25 \%$ capacitance loss after 5000 cycles at $100 \mathrm{mV} \mathrm{s}^{-1}$. The $\mathrm{Ni}_{\mathrm{x}} \mathrm{Co}_{2 \mathrm{x}}(\mathrm{OH})_{6 \mathrm{x}} / \mathrm{TiN}$ electrode outperformed the $\mathrm{NiCo}_{2} \mathrm{O}_{4} / \mathrm{TiN}$ electrode, indicating the advantages of the TiN nanotube arrays as the inner scaffold. 2D inner scaffolds consist of small-sized petals that are the building blocks to construct arrays [163]. Owing to the open space among the building blocks and sometimes their porous nature, the $2 \mathrm{D}$ scaffolds can provide high surface area and facilitate electrolyte to reach the inner part of the electrodes. Xiong et al. prepared graphitic petals (GPs) grown on carbon clothes $(\mathrm{CC})$ as the $2 \mathrm{D}$ scaffold and coated it with a thin layer of polyaniline (PANI), yielding a hybrid CC/GPs/PANI electrode (Figure 13d) [163]. 
The GPs contain a few layers of graphene and naturally offer high surface area and electronic conductivity. The hybrid electrode exhibited a near-theoretical-maximum capacitance for PANI of $2000 \mathrm{~F} \mathrm{~g}^{-1}\left(\sim 230 \mathrm{~F} \mathrm{~cm}^{-3}\right)$ at $1 \mathrm{~A} \mathrm{~g}^{-1}$ and maintained above $1200 \mathrm{~F} \mathrm{~g} \mathrm{~g}^{-1}$ when the current density was as high as $100 \mathrm{~A} \mathrm{~g}^{-1}$. It also delivered a high rate capability with an energy density of $110 \mathrm{Wh} \mathrm{kg}^{-1}$ and a power density of $264 \mathrm{~kW}$ $\mathrm{kg}^{-1}$ at $100 \mathrm{~A} \mathrm{~g}^{-1}$ (Figure 13e). Only 7\% capacitance loss was observed after 2000 cycles (Figure 13f), indicating a long-term cycling stability. Jiang et al. synthesized nullaginite interconnected nanowalls, a type of Ni-based carbonate slat, as precursors to fabricate the "CNTs-pillars-on-nanowall-founation" hybrid structure [164]. The nullaginite nanowalls transform into Ni nanowalls with porous unit nanowall, which is caused by the decomposition of the salt. The Ni nanowalls act as the catalysts to initiate the simultaneous in-situ growth of CNTs on their surface. The unique interconnected hybrid structure and virtues inherited from the conductive CNT network and porous Ni nanowalls were believed to be responsible for the excellent performance.
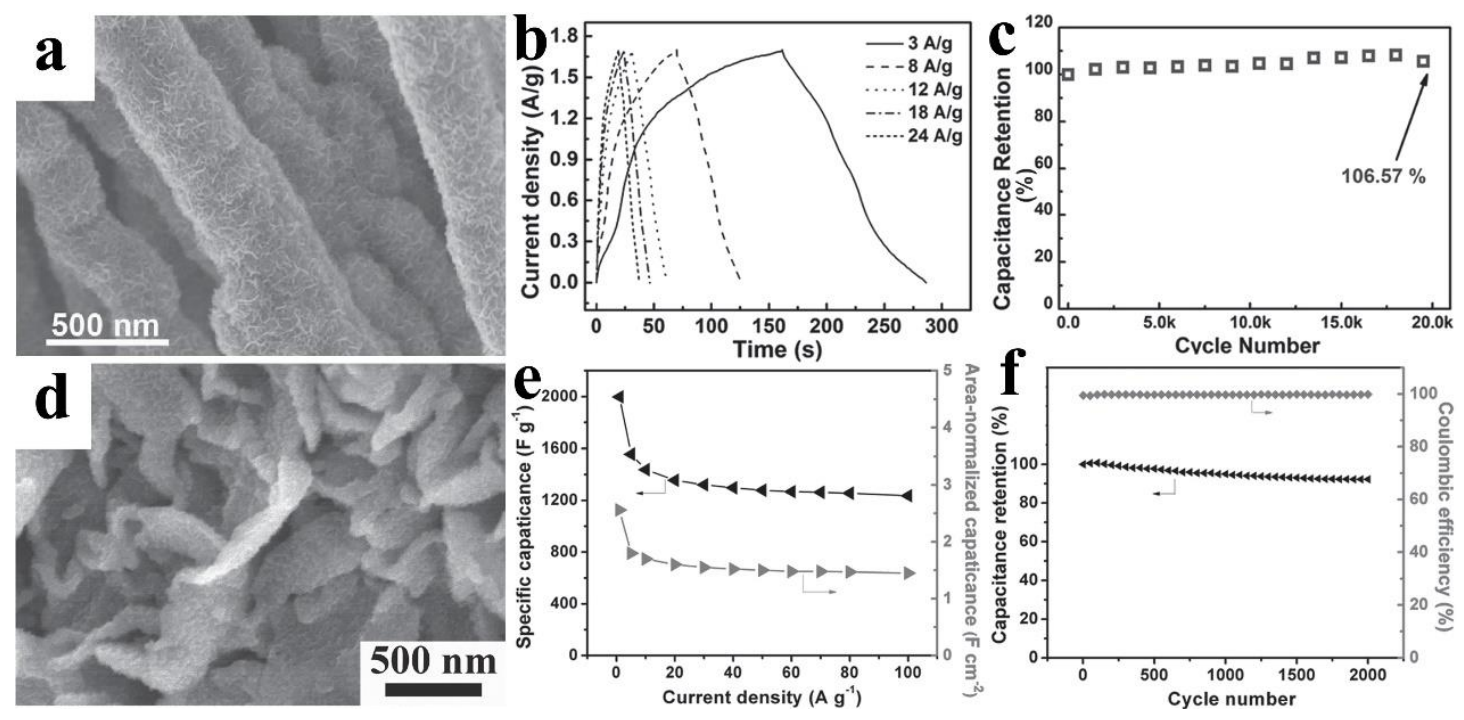

Figure 13. Typical examples of heterogeneous nanostructure arrays with 'FunctionAssistance' working mode for supercapacitor by means of 1D and 2D inner scaffold. (a) Scanning electron microscope (SEM) image of the $\mathrm{Ni}$ nanowire array (NNA)/ $\mathrm{MnO}_{2}$ nanowire arrays. Galvanostatic charging/discharging (GCD) curves (b) 
and cycling performance at a current density of $30 \mathrm{~A} \mathrm{~g}^{-1}$ (c) of NNA-asymmetric micro-supercapacitor (ASC). Reproduced with permission. [161] Copyright 2016, Wiley-VCH. (d) SEM image of conformal polyaniline (PANI) coating on graphite paper (GP) surfaces. Specific capacitances at various current densities (e) and cycling performance at a current density of $10 \mathrm{~mA} \mathrm{~cm}^{-2}$ (f) of the carbon clothes (CC)/GPs/PANI hybrid electrode. Reproduced with permission. [163] Copyright 2013, Wiley-VCH.

Different from 1D and 2D inner scaffold, 3D scaffold shows interconnection of structural units in three dimensions and the resulting pores are in the nanometer scale. The interconnection and nanopores therefore generate large surface area while keeping electron transport pathways in three dimensions. The commonly reported 3D scaffolds include nanoporous metals, 3DOM structures, and 3D porous carbon structures. Nanoporous gold (NPG) fabricated by dealloying Au from Ag-Au alloy shows a bicontinuous structure consisting of quasi-periodic gold ligaments and nanopore channels. The structural features and high conductivity of NPG make it an ideal 3D current collector and thus it has been widely employed for different capacitive materials, including $\mathrm{MnO}_{2}, \mathrm{RuO}_{2}, \mathrm{SnO}_{2}, \mathrm{Ni}(\mathrm{OH})_{2}$, and PPy. For instance, Lang et al. fabricated $\mathrm{NPG} / \mathrm{MnO}_{2}$ heterogeneous electrode where the NPG inner scaffold improves the reaction kinetics of $\mathrm{MnO}_{2}$ thin coating layer (Figure 14a) [165]. The electrode exhibited a capacitance of $1145 \mathrm{~F} \mathrm{~g}^{-1}$ that is close to the theoretical capacitance of $\mathrm{MnO}_{2}\left(\sim 1370 \mathrm{~F} \mathrm{~g} \mathrm{~g}^{-1}\right.$, Figure 14b,14c). The energy and powder densities were reported to be $57 \mathrm{Wh} \mathrm{kg}^{-1}$ and $16 \mathrm{~kW} \mathrm{~kg}^{-1}$, respectively. Owing to the mechanical rigidity and electrochemical stability of the NPG, excellent cycling stability over 30000 cycles was achieved in the case of $\mathrm{SnO}_{2}[166]$ and $\mathrm{Ni}(\mathrm{OH})_{2}$ [167]. Low-cost nanoporous Ni-Mn alloy was reported in the hybrid electrodes with oxy-hydroxides to replace the high-cost gold [168]. 3DOM structures can be prepared by the colloidal crystal templates. Used as inner scaffold, the ordered interconnected pore network of $3 \mathrm{DOM}$ enables rapid ion transport in the electrolyte-filled pores, 
which is advantageous comparing to the circuitous pore structure of the nanoporous metals. However, the application of 3DOM is rather limited due to the multi-step templating methods, and thus has been seen only in $\mathrm{Mn} / \mathrm{MnO}_{2}$ (Figure 14d) [21], $\mathrm{V}_{2} \mathrm{O}_{3} / \mathrm{MnO}_{2}$ [169], $\mathrm{Ni} / \mathrm{Cu}_{2} \mathrm{O}$ [170], and $\mathrm{Ni} / \mathrm{NiO}$ [171]. As an example, Mn 3DOM structure was prepared with ordered polystyrene templates on electrodeposition from an ionic liquid and a 3DOM Mn core- $\mathrm{MnO}_{2}$ shell film was obtained by anodizing the Mn film [21]. A high capacitance of $1200 \mathrm{~F} \mathrm{~g}^{-1}$ was achieved at $5 \mathrm{mV} \mathrm{s}^{-1}$ and $83 \%$ capacitance was maintained when the scan rate increased to $500 \mathrm{mV} \mathrm{s}^{-1}$, indicating a great rate capability of the $3 \mathrm{DOM} \mathrm{Mn} / \mathrm{MnO}_{2}$ core/shell electrode (Figure $14 \mathrm{e}, 14 \mathrm{f}$ ). It is worth noting that, besides high surface area and fast electron transport, fast ion diffusion is also considered as a critical prerequisite for an ideal 3D inner scaffold. As demonstrated in the 3DOM structure, rapid ion transport can be realized by the interconnections among the pores, which highlights the importance of the oriented ion diffusion pathways. In this regard, Zhao et al. reported higher ordered metallic nanopore arrays using a two-step replication through the anodic aluminum oxide (AAO) template-assisted process [172]. The obtained nanopore arrays possess vertically aligned and highly oriented nanopores (Figure 14g), which offers a high orientation to direct ion diffusion. In addition, the surface area of the nanopore arrays can be increased by increasing the aspect-ratio of the starting AAO template, providing feasibility to increase the mass loading of the eletroactive material while keeping rapid ion diffusion and fast electron transport. Using Ni nanopore arrays $/ \mathrm{MnO}_{2}$ as an example (Figure 14h,14i), the authors demonstrated that, when the mass loading increased from 80 to $400 \mu \mathrm{g} \mathrm{cm}^{-2}$, the highly oriented nanopores maintained a pore diameter of about $150 \mathrm{~nm}$ in the hybrid electrode. 

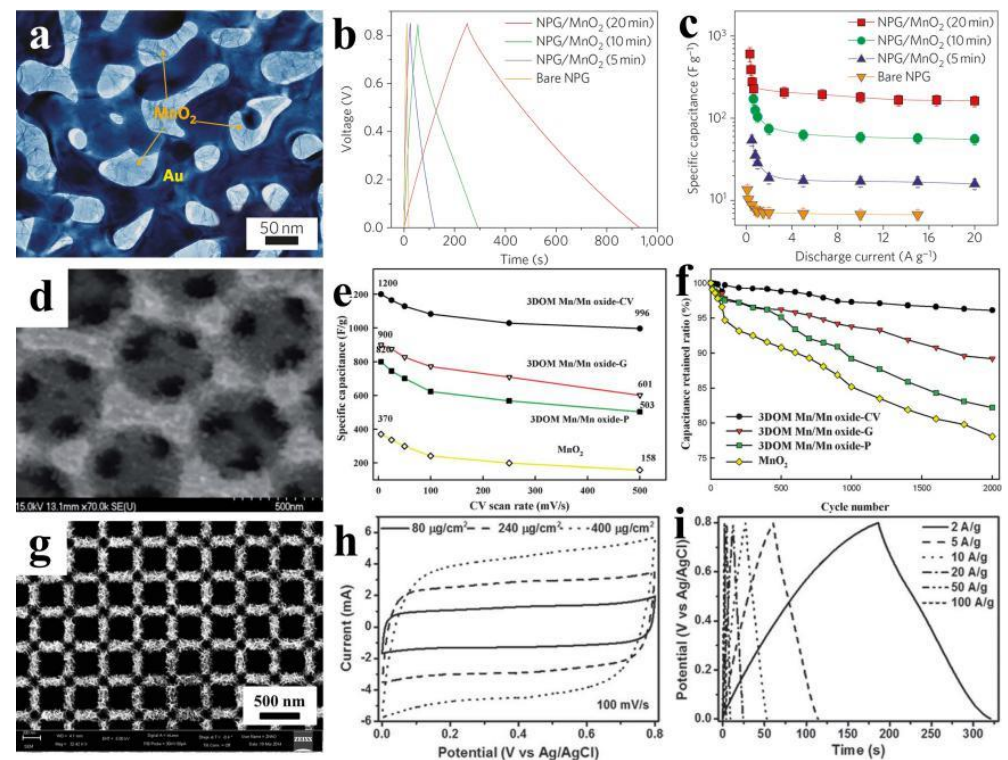

Figure 14. Typical examples of heterogeneous nanostructure arrays with 'FunctionAssistance' working mode for supercapacitor by means of 3D inner scaffold. (a) Bright-field TEM image of the NPA/ $\mathrm{MnO}_{2}$ hybrid with a $\mathrm{MnO}_{2}$ plating time of 20 min. Galvanostatic charging/discharging (GCD) curves at a current density of $0.5 \mathrm{~A}$ $\mathrm{g}^{-1}$ (b) and specific capacitances at various current densities (c) of the hybrids with different $\mathrm{MnO}_{2}$ plating time. Reproduced with permission. [165] Copyright 2011, Springer. (d) SEM image of the $\mathrm{Mn} / \mathrm{MnO}_{2}$ 3DOM structure. Specific capacitances recorded at various scan rates (e) and cycling performance (f) of different $\mathrm{Mn} / \mathrm{MnO}_{2}$ hybrid structures. Reproduced with permission. [21] Copyright 2013, Royal Society of Chemistry. (g) SEM image of the Ni nanopore arrays coated with $\mathrm{MnO}_{2}$. (h) $\mathrm{CV}$ curves of $\mathrm{MnO}_{2} @ \mathrm{Ni}$ nanopore arrays with different $\mathrm{MnO}_{2}$ loadings at a scan rate of $100 \mathrm{mV} \mathrm{s}^{-1}$. (i) GCD curves of the nanopore arrays with a $\mathrm{MnO}_{2}$ loading of $80 \mu \mathrm{g}$ $\mathrm{cm}^{-1}$. Reproduced with permission. [172] Copyright 2014, Wiley-VCH.

\subsubsection{Single-unit device}

Conventional electrochemical supercapacitors store charge in electric double layers or in faradic reactions, permitting large energy density storage on the electrode surface. Power density is however limited because of the requirement for mass transport of ions and/or redox reactions. Electrostatic capacitors can achieve high 
power density because charge can be moved rapidly in a configuration where metal electrodes are separated by a dielectric film and charge is stored on opposing electrode surface. This kind of metal-insulator-metal (MIM) electrostatic capacitor can be prepared in the form of HNAs where each unit structure is a single capacitor, to reach dense packing of active interfaces and thin films. Banerjee et al. demonstrated operational MIM electrostatic nanocapacitor arrays fabricated with ALD inside AAO nanopores, making use of the self-assembly of regular nanopore arrays in AAO together with sequent and multilayer ALD deposition of $\mathrm{TiN}-\mathrm{Al}_{2} \mathrm{O}_{3}$-TiN (Figure 15) [173] [174]. The highly regular arrays have a capacitance per unit planar area of $\sim 10 \mu \mathrm{F} \mathrm{cm} \mathrm{cm}^{-2}$ for $1-\mu \mathrm{m}$-thick $\mathrm{AAO}$ and $\sim 100 \mu \mathrm{F} \mathrm{cm} \mathrm{cm}^{-2}$ for 10- $\mu$ m-thick AAO. The capacitance surpasses equivalent planar capacitance densities of nanoporous structures by a factor of four for the 1- $\mu \mathrm{m}$-deep pores and by a factor of 40 for those that are $10 \mu \mathrm{m}$ deep. The same group detailed nanoengineering of AAO-ALD MIM nanocapacitors using Al doped $\mathrm{ZnO}$ (AZO) as the conducting electrodes [175]. Owing to an intermediate barrier anodic alumina smoothing layer, breakdown fields for the MIM layers increased by 2.5 folds, from about $4 \mathrm{mV} \mathrm{cm}^{-1}$ to about $10 \mathrm{mV} \mathrm{cm}^{-1}$, approaching intrinsic dielectric strength of the $\mathrm{Al}_{2} \mathrm{O}_{3}$ insulator and benefiting to energy density that scales with $V^{2}$.

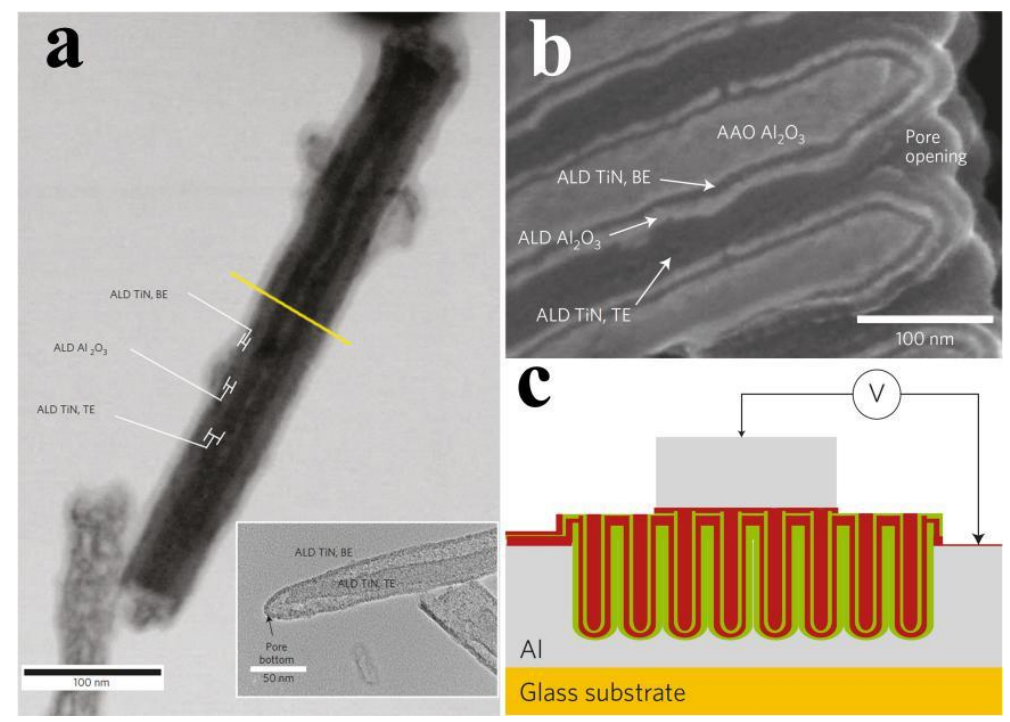

Figure 15. Typical examples of heterogeneous nanostructure arrays with 'Single-unit 
device' working mode for supercapacitor. (a) transmission electron microscope (TEM) image of the MIM capacitors. Inset: the pore bottom with a conformal TiN bottom electrode (BE) and top electrode (TE) coating. (b) SEM image of the metal-insulator-metal (MIM) capacitors, showing a TiN- $\mathrm{Al}_{2} \mathrm{O}_{3}-\mathrm{TiN}$ trilayer structure. (c) Illustration of the electrical testing using the $\mathrm{Al}$ foil (which is in contact with the bottom electrode TiN) as a back contact and electron-beam $\mathrm{Al}$ as the top contact. Reproduced with permission. [173] Copyright 2009, Springer.

\section{Conclusion and perspective}

Heterogenous nanostructure arrays stand as distinguished architectures owing to their distinctive structural features to meet the fundamental challenges concerning generation, transport and utilization of electrons, holes, ions and other molecular species. Hence, Heterogeneous nanostructure arrays have attracted intensive attention and yielded favorable electrochemical performance. Within this context, the motivation of employing heterogeneous nanostructure arrays is present. The variations in size, dimensionality, alignment and constituent offer abundant diversity and resulting superiorities for the applications in electrochemical energy conversion and storage. Three working modes, including 'Function-Function', 'Function-Assistance', 'Single-unit device', take full play of different constituents to realize the synergistic (" $1+1>2$ ") performance through the strong interactions among the constituents.

Despite decades of intense efforts, heterogenous nanostructure arrays in electrochemical energy conversion and storage are still in an early stage. More works are still necessary to achieve impacts to the real world.

First, electrochemical processes taking place either on the surface of or in the bulk of electrode materials could be complex. Direct observations of these processes are necessary to offer "on-site" information that can straightforwardly link to the electrochemical response. In-situ characterization techniques are a perfect solution to obtain such kind of direct observations. Attention should be paid to both 
thermodynamical and dynamic properties. It is vitally important to energy storage devices because their reaction feasibility is associated with dynamic properties while the current in-situ characterizations mostly focus on static studies. In addition, a heterogeneous electrode is often in a $3 \mathrm{D}$ configuration. An in-situ characterization technique that is capable of monitoring the reaction process with respect to a $3 \mathrm{D}$ chemical composition distribution within the electrode is extremely desirable. Cares should be made when developing new in-situ techniques regarding this point.

Second, computational simulations have shown great potential as a powerful tool to understand the electronic structures of the active materials and could be critical to diagnose and design heterostructures. Modeling the heterointerfaces, either solid-solid or solid-liquid, gives us the ability to visualize the local interactions/reactions during the electrochemical processes. It is highly desirable to obtain new insights to the existing mechanisms and even unfold new ones. Simulations could also contribute to develop synthetic routes that are able to realize the designs. Despite we are well-placed to determine the electronic structures, the simulation and understanding of the heterointerfaces are still empirical and tentative, with much references being made on the basis of experimental results. Advanced computational methods must be developed to accurately predict and enable further optimization of the performances of the heterostructures.

Third, design and fabrication of a single heterostructure that embeds all constituents of an electrochemical conversion or storage device is still in the infancy. It remains a great challenge to deliberately incorporate all the constituents in a confined space in a fashion that can empower the device in a maximal degree. We at present are placed to demonstrate the promises of the single-unit devices with the previous reports, but the proposed configurations of the devices are rather limited. New designs are urgently needed to make full use of the demonstrated promises.

In a word, this review focus on the basic working modes of different constituents in heterogeneous nanostructure arrays for electrochemical energy. The attention has been 
paid on not only fundamental science, but also technology of device construction at nanoscale. The basic understanding about the roles of heterogeneous nanostructure arrays may force technological development by producing new requirements to address scientific questions, or by illustrating unconsidered technical possibilities for electrochemical energy conversion and storage. Meanwhile, technology of device construction may in turn benefit for scientific investigations by raising questions about the underlying electrochemical principles. We believe that electrochemical energy conversion and storage devices with high efficiency would be practical in our daily life.

\section{Acknowledgement}

The authors acknowledge funding from European Research Council (ThreeDsurface: 240144), European Research Council (HiNaPc: 737616), BMBF (ZIK-3DNanoDevice: $\quad$ 03Z1MN11), BMBF (Meta-ZIK-BioLithoMorphie: 03Z1M512), and German Research Foundation (DFG: LE 2249_4-1).

\section{Reference}

[1] A.S. Aricò, P. Bruce, B. Scrosati, J.-M. Tarascon, W. van Schalkwijk, Nat. Mater., 4 (2005) 366-377.

[2] Y.-G. Guo, J.-S. Hu, L.-J. Wan, Adv. Mater., 20 (2008) 2878-2887.

[3] P. Yang, J.-M. Tarascon, Nat. Mater., 11 (2012) 560-563.

[4] A.I. Hochbaum, P. Yang, Chem. Rev., 110 (2010) 527-546.

[5] Q. Zhang, E. Uchaker, S.L. Candelaria, G. Cao, Chem. Soc. Rev., 42 (2013) $3127-3171$.

[6] H. Zhao, M. Zhou, L. Wen, Y. Lei, Nano Energy, 13 (2015) 790-813.

[7] J. Liu, G. Cao, Z. Yang, D. Wang, D. Dubois, X. Zhou, G.L. Graff, L.R. Pederson, 
J.-G. Zhang, ChemSusChem, 1 (2008) 676-697.

[8] P. Balaya, Energy Environ. Sci., 1 (2008) 645-654.

[9] K. Rajeshwar, H. Baltruschat, Y.-K. Sun, ChemPhysChem, 15 (2014) 1903-1904.

[10] L. Wen, Z. Wang, Y. Mi, R. Xu, S.-H. Yu, Y. Lei, Small, 11 (2015) 3408-3428.

[11] Y. Xu, M. Zhou, Y. Lei, Adv. Energy Mater., 6 (2016) 1502514.

[12] L. Wen, M. Zhou, C. Wang, Y. Mi, Y. Lei, Adv. Energy Mater., 6 (2016) 1600468.

[13] H. Zheng, Y. Li, H. Liu, X. Yin, Y. Li, Chem. Soc. Rev., 40 (2011) 4506-4524.

[14] M.T. Mayer, Y. Lin, G. Yuan, D. Wang, Acc. Chem. Res., 46 (2013) 1558-1566.

[15] C.X. Kronawitter, L. Vayssieres, S. Shen, L. Guo, D.A. Wheeler, J.Z. Zhang, B.R. Antoun, S.S. Mao, Energy Environ. Sci., 4 (2011) 3889-3899.

[16] A.J. Mieszawska, R. Jalilian, G.U. Sumanasekera, F.P. Zamborini, Small, 3 (2007) $722-756$.

[17] W.S. Jang, S.Y. Kim, J. Lee, J. Park, C.J. Park, C.J. Lee, Chem. Phys. Lett., 422 (2006) 41-45.

[18] Q. Li, C. Wang, J. Am. Chem. Soc., 125 (2003) 9892-9893.

[19] Z. Hongchao, Z. Min, F. Qun, L. Bo, L. Wei, G. Heshuai, W. Minghong, L. Yong, Nanotechnology, 25 (2014) 275603.

[20] W. Zhou, C. Cheng, J. Liu, Y.Y. Tay, J. Jiang, X. Jia, J. Zhang, H. Gong, H.H. Hng, T. Yu, H.J. Fan, Adv. Funct. Mater., 21 (2011) 2439-2445.

[21] M.-J. Deng, P.-J. Ho, C.-Z. Song, S.-A. Chen, J.-F. Lee, J.-M. Chen, K.-T. Lu, Energy Environ. Sci., 6 (2013) 2178-2185.

[22] I.B. Burgess, L. Mishchenko, B.D. Hatton, M. Kolle, M. Lončar, J. Aizenberg, J. Am. Chem. Soc., 133 (2011) 12430-12432. 
[23] Q. Liu, J. He, T. Yao, Z. Sun, W. Cheng, S. He, Y. Xie, Y. Peng, H. Cheng, Y. Sun, Y. Jiang, F. Hu, Z. Xie, W. Yan, Z. Pan, Z. Wu, S. Wei, Nat.Comm., 5 (2014) 5122.

[24] Y. Yang, X. Fan, G. Casillas, Z. Peng, G. Ruan, G. Wang, M.J. Yacaman, J.M. Tour, ACS Nano, 8 (2014) 3939-3946.

[25] S.J. Hurst, E.K. Payne, L. Qin, C.A. Mirkin, Angew. Chem. Int. Ed., 45 (2006) 2672-2692.

[26] C. Guan, X. Wang, Q. Zhang, Z. Fan, H. Zhang, H.J. Fan, Nano Lett., 14 (2014) 4852-4858.

[27] C. Guan, X. Xia, N. Meng, Z. Zeng, X. Cao, C. Soci, H. Zhang, H.J. Fan, Energy Environ. Sci., 5 (2012) 9085-9090.

[28] G. Cai, W. Zhang, L. Jiao, S.-H. Yu, H.-L. Jiang, Chem, 2 (2017) 791-802.

[29] M.-S. Yao, W.-X. Tang, G.-E. Wang, B. Nath, G. Xu, Adv. Mater., 28 (2016) 5229-5234.

[30] Y. Dou, J. Zhou, F. Yang, M.-J. Zhao, Z. Nie, J.-R. Li, J. Mater. Chem. A, 4 (2016) 12526-12534.

[31] X. Qin, Y. Sun, N. Wang, Q. Wei, L. Xie, Y. Xie, J.-R. Li, RSC Adv., 6 (2016) 94177-94183.

[32] L. Wen, R. Xu, Y. Mi, Y. Lei, Nat. Nanotechnol., 12 (2016) 244-250.

[33] A.J. Nozik, Nano Lett., 10 (2010) 2735-2741.

[34] S. Kundu, A. Patra, Chem. Rev., 117 (2017) 712-757.

[35] A.M. Smith, S. Nie, Acc. Chem. Res., 43 (2010) 190-200.

[36] W.E. Buhro, V.L. Colvin, Nat. Mater., 2 (2003) 138-139.

[37] T. Suzuki, H. Watanabe, T. Ueno, Y. Oaki, H. Imai, Langmuir, 33 (2017) 3014-3017. 
[38] A.H. Khan, U. Thupakula, A. Dalui, S. Maji, A. Debangshi, S. Acharya, J. Phys. Chem. C, 117 (2013) 7934-7939.

[39] Y. Sun, Z. Sun, S. Gao, H. Cheng, Q. Liu, F. Lei, S. Wei, Y. Xie, Adv. Energy Mater., 4 (2014) 1300611.

[40] C. Li, J.B. Wright, S. Liu, P. Lu, J.J. Figiel, B. Leung, W.W. Chow, I. Brener, D.D. Koleske, T.-S. Luk, D.F. Feezell, S.R.J. Brueck, G.T. Wang, Nano Lett., 17 (2017) 1049-1055.

[41] K. Kim, J. Choi, T.S. Bae, Appl. Phys. Lett., 90 (2007) 181912.

[42] S.L. Brown, J.B. Miller, R.J. Anthony, U.R. Kortshagen, A. Kryjevski, E.K. Hobbie, ACS Nano, 11 (2017) 1597-1603.

[43] S. Furukawa, T. Miyasato, Phys. Rev. B, 38 (1988) 5726-5729.

[44] R.A. Marcus, Annu. Rev. Phys. Chem., 15 (1964) 155-196.

[45] F.E. Osterloh, Chem. Soc. Rev., 42 (2013) 2294-2320.

[46] M. Zhou, X.W. Lou, Y. Xie, Nano Today, 8 (2013) 598-618.

[47] R. Malik, D. Burch, M. Bazant, G. Ceder, Nano Lett., 10 (2010) 4123-4127.

[48] A.K. Geim, K.S. Novoselov, Nat. Mater., 6 (2007) 183-191.

[49] C.-C. Liu, A.B. Walters, M.A. Vannice, Carbon, 33 (1995) 1699-1708.

[50] M.B. Mohamed, C. Burda, M.A. El-Sayed, Nano Lett., 1 (2001) 589-593.

[51] K.A. Arpin, A. Mihi, H.T. Johnson, A.J. Baca, J.A. Rogers, J.A. Lewis, P.V. Braun, Adv. Mater., 22 (2010) 1084-1101.

[52] P. Poizot, S. Laruelle, S. Grugeon, L. Dupont, J.M. Tarascon, Nature, 407 (2000) 496-499.

[53] Q. Jia, K. Iwashina, A. Kudo, Proc. Natl. Acad. Sci., 109 (2012) 11564-11569. 
[54] J. Su, L. Guo, S. Yoriya, C.A. Grimes, Crystal Grow. Des., 10 (2010) 856-861.

[55] M. Zhou, S. Zhang, Y. Sun, C. Wu, M. Wang, Y. Xie, Chem. Asian J., 5 (2010) 2515-2523.

[56] M. Zhou, J. Bao, Y. Xu, J. Zhang, J. Xie, M. Guan, C. Wang, L. Wen, Y. Lei, Y. Xie, ACS Nano, 8 (2014) 7088-7098.

[57] T.W. Kim, K.-S. Choi, Science, 343 (2014) 990-994.

[58] M. Zhou, H.B. Wu, J. Bao, L. Liang, X.W. Lou, Y. Xie, Angew. Chem. Int. Ed., 52 (2013) 8579-8583.

[59] Y. Xu, E. Memarzadeh Lotfabad, H. Wang, B. Farbod, Z. Xu, A. Kohandehghan, D. Mitlin, Chem. Comm., 49 (2013) 8973-8975.

[60] Y. Xu, M. Zhou, L. Wen, C. Wang, H. Zhao, Y. Mi, L. Liang, Q. Fu, M. Wu, Y. Lei, Chem. Mater., 27 (2015) 4274-4280.

[61] W. Wang, M. Tian, A. Abdulagatov, S.M. George, Y.-C. Lee, R. Yang, Nano Lett., 12 (2012) 655-660.

[62] J.S. Chen, Y.L. Tan, C.M. Li, Y.L. Cheah, D. Luan, S. Madhavi, F.Y.C. Boey, L.A. Archer, X.W. Lou, J. Am. Chem. Soc., 132 (2010) 6124-6130.

[63] S. Liu, H. Jia, L. Han, J. Wang, P. Gao, D. Xu, J. Yang, S. Che, Adv. Mater., 24 (2012) 3201-3204.

[64] M. Zhou, Y. Xu, C. Wang, Q. Li, J. Xiang, L. Liang, M. Wu, H. Zhao, Y. Lei, Nano Energy, 31 (2017) 514-524.

[65] M. Zhou, Y. Xu, J. Xiang, C. Wang, L. Liang, L. Wen, Y. Fang, Y. Mi, Y. Lei, Adv. Energy Mater., 6 (2016) 1600448-n/a.

[66] M. Zhou, J. Bao, M. Tao, R. Zhu, Y. Zeng, Z. Wei, Y. Xie, Chem. Comm., 48 (2012) 3439-3441. 
[67] M. Woodhouse, B.A. Parkinson, Chem. Soc. Rev., 38 (2009) 197-210.

[68] R. Liu, Y. Lin, L.-Y. Chou, S.W. Sheehan, W. He, F. Zhang, H.J.M. Hou, D. Wang, Angew. Chem. Int. Ed., 50 (2011) 499-502.

[69] M.P. Dare-Edwards, J.B. Goodenough, A. Hamnett, P.R. Trevellick, J. Chem. Soc., Faraday Trans. 1,, 79 (1983) 2027-2041.

[70] S. Zhu, D. Wang, Adv. Energy Mater., 60 (2017) 1700841.

[71] S.V. Eliseeva, J.-C.G. Bunzli, New J. Chem., 35 (2011) 1165-1176.

[72] M.G. Walter, E.L. Warren, J.R. McKone, S.W. Boettcher, Q. Mi, E.A. Santori, N.S. Lewis, Chem. Rev., 110 (2010) 6446-6473.

[73] C. Dette, M.A. Pérez-Osorio, C.S. Kley, P. Punke, C.E. Patrick, P. Jacobson, F. Giustino, S.J. Jung, K. Kern, Nano Lett., 14 (2014) 6533-6538.

[74] A. Al-Haddad, Z. Wang, M. Zhou, S. Tarish, R. Vellacheri, Y. Lei, Small, 12 (2016) 5538-5542.

[75] M.T. Mayer, C. Du, D. Wang, J. Am. Chem. Soc., 134 (2012) 12406-12409.

[76] S.K. Karuturi, C. Cheng, L. Liu, L. Tat Su, H.J. Fan, A.I.Y. Tok, Nano Energy, 1 (2012) 322-327.

[77] S.K. Karuturi, J. Luo, C. Cheng, L. Liu, L.T. Su, A.I.Y. Tok, H.J. Fan, Adv. Mater., 24 (2012) 4157-4162.

[78] W. Sheng, B. Sun, T. Shi, X. Tan, Z. Peng, G. Liao, ACS Nano, 8 (2014) 7163-7169.

[79] Y.J. Hwang, A. Boukai, P. Yang, Nano Lett., 9 (2009) 410-415.

[80] J. Shi, Y. Hara, C. Sun, M.A. Anderson, X. Wang, Nano Lett., 11 (2011) 3413-3419.

[81] X. Qi, G. She, X. Huang, T. Zhang, H. Wang, L. Mu, W. Shi, Nanoscale, 6 (2014) 
3182-3189.

[82] Y. Gun, G.Y. Song, V.H.V. Quy, J. Heo, H. Lee, K.-S. Ahn, S.H. Kang, ACS Appl. Mater. Inter., 7 (2015) 20292-20303.

[83] S. Hernández, V. Cauda, A. Chiodoni, S. Dallorto, A. Sacco, D. Hidalgo, E. Celasco, C.F. Pirri, ACS Appl. Mater. Inter., 6 (2014) 12153-12167.

[84] W. Smith, A. Wolcott, R.C. Fitzmorris, J.Z. Zhang, Y. Zhao, J. Mater. Chem., 21 (2011) 10792-10800.

[85] Y. Li, R. Wang, H. Li, X. Wei, J. Feng, K. Liu, Y. Dang, A. Zhou, J. Phys. Chem. C, 119 (2015) 20283-20292.

[86] J. Luo, L. Ma, T. He, C.F. Ng, S. Wang, H. Sun, H.J. Fan, J. Phys. Chem. C, 116 (2012) 11956-11963.

[87] Y. Pi, Z. Li, D. Xu, J. Liu, Y. Li, F. Zhang, G. Zhang, W. Peng, X. Fan, ACS Sustain. Chem. Eng., 5 (2017) 5175-5182.

[88] A. Mumtaz, N.M. Mohamed, M. Mazhar, M.A. Ehsan, M.S. Mohamed Saheed, ACS Appl. Mater. Inter., 8 (2016) 9037-9049.

[89] M. Zalfani, B. van der Schueren, Z.-Y. Hu, J.C. Rooke, R. Bourguiga, M. Wu, Y. Li, G. Van Tendeloo, B.-L. Su, J. Mater. Chem. A, 3 (2015) 21244-21256.

[90] A. Sarkar, A.K. Singh, D. Sarkar, G.G. Khan, K. Mandal, ACS Sustain. Chem. Eng., 3 (2015) 2254-2263.

[91] G. Yun, M. Balamurugan, H.-S. Kim, K.-S. Ahn, S.H. Kang, J. Phys. Chem. C, 120 (2016) 5906-5915.

[92] H.M. Chen, C.K. Chen, R.-S. Liu, C.-C. Wu, W.-S. Chang, K.-H. Chen, T.-S. Chan, J.-F. Lee, D.P. Tsai, Adv. Energy Mater., 1 (2011) 742-747.

[93] Y. Pihosh, I. Turkevych, K. Mawatari, J. Uemura, Y. Kazoe, S. Kosar, K. Makita, T. Sugaya, T. Matsui, D. Fujita, M. Tosa, M. Kondo, T. Kitamori, Sc. Rep., 5 (2015) 
11141.

[94] J. Su, L. Guo, N. Bao, C.A. Grimes, Nano Lett., 11 (2011) 1928-1933.

[95] M. Balamurugan, G. Yun, K.-S. Ahn, S.H. Kang, J. Phys. Chem. C, 121 (2017) 7625-7634.

[96] L. Caccamo, J. Hartmann, C. Fàbrega, S. Estradé, G. Lilienkamp, J.D. Prades, M.W.G. Hoffmann, J. Ledig, A. Wagner, X. Wang, L. Lopez-Conesa, F. Peiró, J.M. Rebled, H.-H. Wehmann, W. Daum, H. Shen, A. Waag, ACS Appl. Mater. Inter., 6 (2014) 2235-2240.

[97] L.T. Su, S.K. Karuturi, J. Luo, L. Liu, X. Liu, J. Guo, T.C. Sum, R. Deng, H.J. Fan, X. Liu, A.I.Y. Tok, Adv. Mater., 25 (2013) 1603-1607.

[98] Y. Tang, W. Di, X. Zhai, R. Yang, W. Qin, ACS Cata., 3 (2013) 405-412.

[99] K.-H. Ye, J.-Y. Wang, N. Li, Z.-Q. Liu, S.-H. Guo, Y.-P. Guo, Y.-Z. Su, Inor. Chem. Comm., 45 (2014) 116-119.

[100] X. Zhang, H. Huang, J. Liu, Y. Liu, Z. Kang, J. Mater. Chem. A, 1 (2013) 11529-11533.

[101] C. Gao, Q. Zhang, Z. Lu, Y. Yin, J. Am. Chem. Soc., 133 (2011) 19706-19709.

[102] G. Wang, S. Tao, Y. Liu, L. Guo, G. Qin, K. Ijiro, M. Maeda, Y. Yin, Chem. Comm., 52 (2016) 398-401.

[103] M. Wang, C. Gao, L. He, Q. Lu, J. Zhang, C. Tang, S. Zorba, Y. Yin, J. Am. Chem. Soc., 135 (2013) 15302-15305.

[104] S. Linic, P. Christopher, D.B. Ingram, Nat. Mater., 10 (2011) 911.

[105] X. Zheng, L. Zhang, Energy Environ. Sci., 9 (2016) 2511-2532.

[106] R. Xu, L. Wen, Z. Wang, H. Zhao, S. Xu, Y. Mi, Y. Xu, M. Sommerfeld, Y. Fang, Y. Lei, ACS Nano, 11 (2017) 7382-7389. 
[107] S.C. Warren, E. Thimsen, Energy Environ. Sci., 5 (2012) 5133-5146.

[108] Z. Zhan, Y. Lei, ACS Nano, 8 (2014) 3862-3868.

[109] Z. Wang, D. Cao, L. Wen, R. Xu, M. Obergfell, Y. Mi, Z. Zhan, N. Nasori, J. Demsar, Y. Lei, Nat. Commun., 7 (2016) 10348.

[110] J. Li, S.K. Cushing, P. Zheng, F. Meng, D. Chu, N. Wu, Nat. Commun., 4 (2013) 2651.

[111] J. Li, Y. Qiu, Z. Wei, Q. Lin, Q. Zhang, K. Yan, H. Chen, S. Xiao, Z. Fan, S. Yang, Energy Environ. Sci., 7 (2014) 3651-3658.

[112] Y. Qiu, S.-F. Leung, Q. Zhang, B. Hua, Q. Lin, Z. Wei, K.-H. Tsui, Y. Zhang, S. Yang, Z. Fan, Nano Lett., 14 (2014) 2123-2129.

[113] J. Lee, S. Mubeen, X. Ji, G.D. Stucky, M. Moskovits, Nano Lett., 12 (2012) 5014-5019.

[114] H. Eom, J.-Y. Jung, Y. Shin, S. Kim, J.-H. Choi, E. Lee, J.-H. Jeong, I. Park, Nanoscale, 6 (2014) 226-234.

[115] X. Wang, R. Long, D. Liu, D. Yang, C. Wang, Y. Xiong, Nano Energy, 24 (2016) 87-93.

[116] X. Zhang, Y. Liu, S.-T. Lee, S. Yang, Z. Kang, Energy Environ. Sci., 7 (2014) 1409-1419.

[117] J. Li, S.K. Cushing, P. Zheng, T. Senty, F. Meng, A.D. Bristow, A. Manivannan, N. Wu, J. Am. Chem. Soc., 136 (2014) 8438-8449.

[118] L. Zhang, C.-Y. Lin, V.K. Valev, E. Reisner, U. Steiner, J.J. Baumberg, Small, 10 (2014) 3970-3978.

[119] X. Wang, C. Liow, A. Bisht, X. Liu, T.C. Sum, X. Chen, S. Li, Adv. Mater., 27 (2015) 2207-2214. 
[120] Z.W. Seh, S. Liu, M. Low, S.-Y. Zhang, Z. Liu, A. Mlayah, M.-Y. Han, Adv. Mater., 24 (2012) 2310-2314.

[121] X. Wang, C. Liow, D. Qi, B. Zhu, W.R. Leow, H. Wang, C. Xue, X. Chen, S. Li, Adv. Mater., 26 (2014) 3506-3512.

[122] F. Wu, Y. Yu, H. Yang, L.N. German, Z. Li, J. Chen, W. Yang, L. Huang, W. Shi, L. Wang, X. Wang, Adv. Mater., 29 (2017) 1701432.

[123] Y. Mi, L. Wen, R. Xu, Z. Wang, D. Cao, Y. Fang, Y. Lei, Advanced Energy Materials, 6 (2016) 1501496.

[124] Y. Lin, S. Zhou, X. Liu, S. Sheehan, D. Wang, J. Am. Chem. Soc., 131 (2009) 2772-2773.

[125] Y. Lin, S. Zhou, S.W. Sheehan, D. Wang, J. Am. Chem. Soc., 133 (2011) 2398-2401.

[126] C.Y. Cummings, F. Marken, L.M. Peter, A.A. Tahir, K.G.U. Wijayantha, Chem. Comm., 48 (2012) 2027-2029.

[127] M. Zhou, J. Bao, W. Bi, Y. Zeng, R. Zhu, M. Tao, Y. Xie, ChemSusChem, 5 (2012) 1420-1425.

[128] Y. He, P. Ma, S. Zhu, M. Liu, Q. Dong, J. Espano, X. Yao, D. Wang, Joule, 1 (2017) 831-842.

[129] F. Le Formal, N. Tetreault, M. Cornuz, T. Moehl, M. Gratzel, K. Sivula, Chem. Sci., 2 (2011) 737-743.

[130] A. Paracchino, V. Laporte, K. Sivula, M. Grätzel, E. Thimsen, Nat. Mater., 10 (2011) 456-461.

[131] Y. He, James E. Thorne, Cheng H. Wu, P. Ma, C. Du, Q. Dong, J. Guo, D. Wang, Chem, 1 (2016) 640-655.

[132] Y. Yu, Z. Zhang, X. Yin, A. Kvit, Q. Liao, Z. Kang, X. Yan, Y. Zhang, X. Wang, 
Nat. Energy, 2 (2017) 17045.

[133] S. Mubeen, N. Singh, J. Lee, G.D. Stucky, M. Moskovits, E.W. McFarland, Nano Lett., 13 (2013) 2110-2115.

[134] S. Mubeen, J. Lee, N. Singh, S. Krämer, G.D. Stucky, M. Moskovits, Nat. Nanotechnol., 8 (2013) 247-251.

[135] C. Liu, J. Tang, H.M. Chen, B. Liu, P. Yang, Nano Lett., 13 (2013) 2989-2992.

[136] T. Song, H. Cheng, H. Choi, J.-H. Lee, H. Han, D.H. Lee, D.S. Yoo, M.-S. Kwon, J.-M. Choi, S.G. Doo, H. Chang, J. Xiao, Y. Huang, W.I. Park, Y.-C. Chung, H. Kim, J.A. Rogers, U. Paik, ACS Nano, 6 (2012) 303-309.

[137] J. Wang, Q. Zhang, X. Li, D. Xu, Z. Wang, H. Guo, K. Zhang, Nano Energy, 6 (2014) 19-26.

[138] D. Kong, C. Cheng, Y. Wang, Z. Huang, B. Liu, Y. Von Lim, Q. Ge, H.Y. Yang, J. Mater. Chem. A, 5 (2017) 9122-9131.

[139] S. Zhou, X. Yang, Y. Lin, J. Xie, D. Wang, ACS Nano, 6 (2012) 919-924.

[140] H. Zhang, X. Yu, P.V. Braun, Nat. Nanotechnol., 6 (2011) 277.

[141] C. Chen, Y. Zhang, Y. Li, Y. Kuang, J. Song, W. Luo, Y. Wang, Y. Yao, G. Pastel, J. Xie, L. Hu, Adv. Energy Mater., 7 (2017) 1700595.

[142] X. Xie, K. Kretschmer, J. Zhang, B. Sun, D. Su, G. Wang, Nano Energy, 13 (2015) 208-217.

[143] G. Tan, F. Wu, Y. Yuan, R. Chen, T. Zhao, Y. Yao, J. Qian, J. Liu, Y. Ye, R. Shahbazian-Yassar, J. Lu, K. Amine, Nat. Commun.,, 7 (2016) 11774.

[144] Y. Yao, N. Liu, M.T. McDowell, M. Pasta, Y. Cui, Energy Environ. Sci., 5 (2012) 7927-7930.

[145] Y. Zhong, Y. Ma, Q. Guo, J. Liu, Y. Wang, M. Yang, H. Xia, Sci. Rep., 7 (2017) 
40927.

[146] J. Guo, Y. Cai, S. Zhang, S. Chen, F. Zhang, ACS Appl. Mater. Inter., 8 (2016) 16116-16124.

[147] J. Liu, N. Li, M.D. Goodman, H.G. Zhang, E.S. Epstein, B. Huang, Z. Pan, J. Kim, J.H. Choi, X. Huang, J. Liu, K.J. Hsia, S.J. Dillon, P.V. Braun, ACS Nano, 9 (2015) 1985-1994.

[148] Y. Liu, Y. Xu, Y. Zhu, J.N. Culver, C.A. Lundgren, K. Xu, C. Wang, ACS Nano, 7 (2013) 3627-3634.

[149] C. Liu, E.I. Gillette, X. Chen, A.J. Pearse, A.C. Kozen, M.A. Schroeder, K.E. Gregorczyk, S.B. Lee, G.W. Rubloff, Nat. Nanotechnol., 9 (2014) 1031-1039.

[150] S.R. Gowda, A. Leela Mohana Reddy, X. Zhan, P.M. Ajayan, Nano Lett., 11 (2011) 3329-3333.

[151] N. Choudhary, C. Li, J. Moore, N. Nagaiah, L. Zhai, Y. Jung, J. Thomas, Adv. Mater., 29 (2017) 1605336.

[152] B.C. Kim, J.-Y. Hong, G.G. Wallace, H.S. Park, Adv. Energy Mater., 5 (2015) 1500959.

[153] Y. Xiang, S. Lu, S.P. Jiang, Chem. Soc. Rev., 41 (2012) 7291-7321.

[154] Z. Yang, J. Zhang, M.C.W. Kintner-Meyer, X. Lu, D. Choi, J.P. Lemmon, J. Liu, Chem. Rev., 111 (2011) 3577-3613.

[155] X. Xia, J. Tu, Y. Zhang, X. Wang, C. Gu, X.-b. Zhao, H.J. Fan, ACS Nano, 6 (2012) 5531-5538.

[156] C. Zhu, L. Yang, J.K. Seo, X. Zhang, S. Wang, J. Shin, D. Chao, H. Zhang, Y.S. Meng, H.J. Fan, Mater. Horiz., 4 (2017) 415-422.

[157] J. Xiao, L. Wan, S. Yang, F. Xiao, S. Wang, Nano Lett., 14 (2014) 831-838. 
[158] W. Fu, C. Zhao, W. Han, Y. Liu, H. Zhao, Y. Ma, E. Xie, J. Mater. Chem. A, 3 (2015) 10492-10497.

[159] C. Zhou, Y. Zhang, Y. Li, J. Liu, Nano Lett., 13 (2013) 2078-2085.

[160] Y. Zeng, Y. Han, Y. Zhao, Y. Zeng, M. Yu, Y. Liu, H. Tang, Y. Tong, X. Lu, Adv. Energy Mater., 5 (2015) 1402176.

[161] C. Xu, Z. Li, C. Yang, P. Zou, B. Xie, Z. Lin, Z. Zhang, B. Li, F. Kang, C.-P. Wong, Adv. Mater., 28 (2016) 4105-4110.

[162] F. Grote, Y. Lei, Nano Energy, 10 (2014) 63-70.

[163] G. Xiong, C. Meng, R.G. Reifenberger, P.P. Irazoqui, T.S. Fisher, Adv. Energy Mater., 4 (2014) 1300515.

[164] J. Jiang, J. Liu, W. Zhou, J. Zhu, X. Huang, X. Qi, H. Zhang, T. Yu, Energy Environ. Sci., 4 (2011) 5000-5007.

[165] X. Lang, A. Hirata, T. Fujita, M. Chen, Nat. Nanotechnol., 6 (2011) 232.

[166] X. Lang, A. Hirata, T. Fujita, M. Chen, Adv. Energy Mater., 4 (2014) 1301809.

[167] S.-I. Kim, S.-W. Kim, K. Jung, J.-B. Kim, J.-H. Jang, Nano Energy, 24 (2016) $17-24$.

[168] J. Kang, A. Hirata, H.J. Qiu, L. Chen, X. Ge, T. Fujita, M. Chen, Adv. Mater., 26 (2014) 269-272.

[169] X.-Y. Lang, B.-T. Liu, X.-M. Shi, Y.-Q. Li, Z. Wen, Q. Jiang, Adv. Sci., 3 (2016) 1500319 .

[170] M.-J. Deng, C.-Z. Song, P.-J. Ho, C.-C. Wang, J.-M. Chen, K.-T. Lu, Phys. Chem. Chem. Phys., 15 (2013) 7479-7483.

[171] J.-H. Kim, S.H. Kang, K. Zhu, J.Y. Kim, N.R. Neale, A.J. Frank, Chem. Commun., 47 (2011) 5214-5216. 
[172] H. Zhao, C. Wang, R. Vellacheri, M. Zhou, Y. Xu, Q. Fu, M. Wu, F. Grote, Y. Lei, Adv. Mater., 26 (2014) 7654-7659.

[173] P. Banerjee, I. Perez, L. Henn-Lecordier, S.B. Lee, G.W. Rubloff, Nat. Nanotechnol., 4 (2009) 292.

[174] P. Banerjee, I. Perez, L. Henn-Lecordier, S.B. Lee, G.W. Rubloff, ECS Trans., 25 (2009) 345-353.

[175] L.C. Haspert, S.B. Lee, G.W. Rubloff, ACS Nano, 6 (2012) 3528-3536. 